\title{
On Hansen's Version of Spectral Theory and the Moyal Product
}

\author{
By \\ Mark A. Hennings* and Daniel A. Dubin**
}

\section{$\S 1 . \quad$ Introduction}

\section{§1.1. A brief history of the Moyal product}

The story of the Moyal product begins in 1927 with Weyl's introduction of the unitary operators ${ }^{1}$ associated with position and momentum in the Schrödinger representation, and more particularly with the collection of unitary operators $W(a, b)=\exp i(a P+b Q)(a, b \in \mathbb{R})[27,28]$. If $f \in L^{1}(E)$ is an integrable function on phase space $E=\mathbb{R}^{2}$, the operator $W(f)=\int f(x) W(x) d^{2} x$ can be defined. In $[18,19]$ von Neumann gave an explicit formula for the product $f \circ g$ defined by a $W(f \circ g)=W(f) W(g)$ for $f, g \in L^{1}(E)$. This product $f \circ g$ is now known as the twisted convolution of $f$ and $g$. Then the formula

$$
\Delta[f]=\frac{1}{2 \pi} W(\mathcal{F} f)
$$

defines the Weyl quantization of the function $f$ (here $\mathcal{F}$ denotes the Fourier transform). Associated with Weyl quantization is the Moyal product $f \star g=$ $\frac{1}{2 \pi} \mathcal{F} f \circ \mathcal{F} g$, so that $\Delta[f \star g]=\Delta[f] \Delta[g]$.

This formalism was further developed by Wigner in 1932, [29], who introduced what is known as the Wigner transform. Inter alia, this transform permits a more rigorous handling of the formalisms of Weyl quantization. The

\footnotetext{
Communicated by M. Kashiwara. Received September 19, 2008. Revised February 21, 2009.

2000 Mathematics Subject Classification(s): Primary: 81S10, Secondary: 81S30, 46Fxx, 46H30, 47A10, 47N50.

*Rugby School, Rugby, Warwickshire, CV22 5EH.

e-mail: MAH@rugbyschool.net

** Clarendon Laboratory, University of Oxford, OX1 3PU.

e-mail: d.dubin1@physics.ox.ac.uk

${ }^{1}$ The construction outlined here is for one degree of freedom; extension to $d$ degrees of freedom is trivial.
}

(C) 2009 Research Institute for Mathematical Sciences, Kyoto University. All rights reserved. 
next important development was due to Moyal in 1949, [17]. While exploring the statistical aspects of quantum mechanics, he introduced the Moyal bracket $\{f \star g\}$ in operator form. However he did not connect his bracket with the commutator expression $f \star g-g \star f$. Nor did he notice that the Weyl quantization of $\{f \star g\}$ was equal to the operator commutator of $\Delta[f]$ and $\Delta[g]$.

Thus the basic structure for considering the phase space correlate of quantum mechanics was known, but much detail was lacking. In particular there was no concern for the precise classes of functions and distributions to which the formalism held, nor a mathematically rigorous analysis of the semi-classical regime, nor any development of the geometry inherent in this structure.

Some more detailed analysis was provided by Pool, [20], who showed (with appropriate structure and complete rigour) that Weyl quantization provides a unitary correspondence between the space $L^{2}(E)$ and the space of HilbertSchmidt operators on Hilbert space. Once this was available, the way was open to embed the Pool structure into distribution theory ${ }^{2}$ (once this became generally available after 1959, [23]) and hence to begin to consider a wider class of operators, in particular, unbounded operators.

The fundamental problem extending the Moyal product to distributions is that it cannot be defined on all distributions. Various classes of distributions were considered. For example, Antonets, [2], considered the ${ }^{*}$-algebra $\mathcal{W}_{\hbar}$ of *-multipliers of the test functions (we shall denote this space as $N \cap \bar{N}$ in this paper). These are the distributions whose Weyl quantizations are (continuous) endomorphisms of Schwartz space with adjoints which are also such endomorphisms. In a different direction, Daubechies, [3], considered the class $D_{b}^{\prime}$ of distributions whose Weyl quantizations are bounded, and also the class of distributions with trace-class quantizations. Of course, $D_{b}^{\prime}$ contains the whole space $L^{2}(E)$ studied by Pool, but there are several interesting distributions on phase space (such as position and momentum!) which are not bounded. On the other hand, $N \cap \bar{N}$ is a large space of distributions containing many important distributions. Moreover, there is a considerable overlap between $N \cap \bar{N}$ and $D_{b}^{\prime}$, [7], but $N \cap \bar{N}$ still does not contain the whole of $L^{2}(E)$. For other work applying functional analysis to the phase space formalism, see [26, 12, 13, 21, 24].

This is a typical obstruction in the theory: whenever one improves one property, something else is spoiled. In this paper, for example, we shall extend both spaces $N \cap \bar{N}$ and $D_{b}^{\prime}$ to a larger space $Q \cap \bar{Q}$ on which it is possible to define a Moyal product; the downside is that with respect to this Moyal

\footnotetext{
${ }^{2}$ Throughout this article, distributions will be tempered, and so test functions will be $\mathcal{S}$-class.
} 
product $Q \cap \bar{Q}$ is only a partial *-algebra, [1].

There have been other approaches to the Moyal product to the one we intend to consider. The reason for our focusing on the above formalism lies in our wanting to consider the possibility of a spectral theory associated with the Moyal product. In spectral theory, properties such as (essential) self-adjointness are key. For that reason, the Kohn-Nirenberg calculus $[11,14,16]$ of pseudodifferential operators (while producing a phase space product similar to the Moyal product) will not be considered in detail. Nor shall we be concerned with the deformation approach to quantization, [8, 15, 21], although much interesting mathematics and physics can be derived from it. This formulates the formulae of quantum mechanics as series expansions in Planck's constant $\hbar$, and derives important information by considering asymptotic expansions as $\hbar \rightarrow 0$. In this sense, the Moyal product is seen as a deformation of the pointwise product in phase space, while the Moyal bracket is a deformation of the Poisson bracket.

The main burden of this paper is concerned with previous work by Hansen, [9], who proposed a method for transforming spectral theory from operators on Hilbert space to the distributions on phase space themselves. This is a new departure from the work of earlier authors, but a very interesting one, since the key idea of the Moyal product is to encapsulate the structure of quantum mechanics within phase space. Consequently, it should be possible to understand the spectral theory of the Weyl quantization of a distribution in terms of properties of that distribution itself. Whether a distribution's quantization is essentially self-adjoint should be a question that can be answered within distribution theory, and the resolvent of a self-adjoint operator should be expressible in terms of suitable distributions.

Now the phase space formalism will be effective in this regard only when the Moyal product is extended to products of distributions. It is both evident and provable that it is only extendible to pairs of distributions belonging to certain classes, for example Hörmander's $S_{\rho, \delta}^{m}$ class [10]. It was Hansen's suggestion that it was possible to extend the Moyal product sufficiently so that one could effectively determine a spectral representation for a symmetric, but not necessarily self adjoint, operator (and other classes of operators as well).

This would be a serious short-cut in spectral theory, certainly a considerable advance, but as we shall show below, Hansen's work assumed an unjustified associativity for a certain partial *-algebra. At the expense of considerable technical analysis, we push Hansen's programme on, without the associativity assumption. But we do not obtain any short-cut in spectral theory. If anything, 
the resulting analysis is considerably less practicable. Nonetheless there is hope that better designed classes of distributions might move things forward. In any event, in our opinion the work of Hansen is sufficiently original that it deserves working through without the associativity assumption, even at the expense of the length of this paper.

\section{$\S 1.2$. Technical preview}

The structure of the Moyal product on $\mathcal{S}\left(\mathbb{R}^{2 d}\right)$ is such that there is a subspace isomorphic to $\mathcal{S}\left(\mathbb{R}^{d}\right)$ which is invariant under multipliers ${ }^{3}$. Thus any multiplier on $\mathcal{S}\left(\mathbb{R}^{2 d}\right)$ can be regarded as an operator on $\mathcal{S}\left(\mathbb{R}^{d}\right)$, and so we have a system whereby functions and distributions on phase space $\mathbb{R}^{2 d}$ can be regarded as operators on $\mathcal{S}\left(\mathbb{R}^{d}\right)$. It is claimed by Hansen, [9], that operators of this type which are symmetric possess a distribution-valued resolvent map which permits an associated spectral resolution. As noted above, there are technical problems with this statement that need to be resolved, and it is the purpose of this paper to discuss them.

The method in question first uses the Moyal product to define a twisted convolution on Schwartz space $\mathcal{S}\left(\mathbb{R}^{2 d}\right)$, and then introduces a familiar and usual extension whereby the twisted convolution of a tempered distribution in $\mathcal{S}^{\prime}\left(\mathbb{R}^{2 d}\right)$ and an element of Schwartz space $\mathcal{S}\left(\mathbb{R}^{2 d}\right)$ can be defined (as a continuous product). The resulting quantity is, in general, a tempered distribution, but it is possible to restrict attention to those elements of $\mathcal{S}^{\prime}\left(\mathbb{R}^{2 d}\right)$ whose convolution with a Schwartz function is always a Schwartz function ${ }^{4}$. This is (almost) the $\mathcal{W}_{\hbar}$ space of Antonets, [2]. Such tempered distributions then generate (continuous) endomorphisms of $\mathcal{S}\left(\mathbb{R}^{2 d}\right)$ which act as multipliers, and hence (by restriction) continuous endomorphisms of $\mathcal{S}\left(\mathbb{R}^{d}\right)$. Composing the endomorphisms (either of $\mathcal{S}\left(\mathbb{R}^{2 d}\right)$ or of $\mathcal{S}\left(\mathbb{R}^{d}\right)$ ) corresponding to two of these distributions results in another such endomorphism, which can then be associated with a tempered distribution. If this final distribution is called the Moyal product of the two original distributions, then the Moyal product on $\mathcal{S}\left(\mathbb{R}^{2 d}\right)$ has been extended to define a structure of an associative algebra on this class of tempered distributions. Moreover, it is possible to define a suitable involution on this algebra, and it is sensible to restrict out attention to those distributions which correspond to endomorphisms of $\mathcal{S}\left(\mathbb{R}^{2 d}\right)$ whose adjoints are also endomorphisms of $\mathcal{S}\left(\mathbb{R}^{2 d}\right)$. In other words, we choose to consider only

\footnotetext{
${ }^{3}$ The terms multiplier and product will refer to the Moyal product unless otherwise specified.

${ }^{4} \mathcal{S}\left(\mathbb{R}^{2 d}\right)$ is naturally a subspace of $\mathcal{S}^{\prime}\left(\mathbb{R}^{2 d}\right)$.
} 
those tempered distributions which correspond to elements of $\mathcal{L}^{+}\left(\mathcal{S}\left(\mathbb{R}^{2 d}\right)\right)$ (or, by restriction, to $\left.\mathcal{L}^{+}\left(\mathcal{S}\left(\mathbb{R}^{d}\right)\right)\right)$. So far as this goes, there is no problem.

However, this class of distributions is not large enough to support the study of the spectral properties of the associated operators. Thus we are forced to work with distributions whose twisted convolution with a Schwartz function is no longer a Schwartz function, and this means that we cannot always form the Moyal product of two distributions. What we have is a partial *-algebra of distributions, with all the problems of non-associativity of the product (when it exists) that entails. Some of the stronger claims to be found in [9] seem to arise from assumptions of associativity for this partial *-algebra.

Our technique thus extends the work of Antonets, but must be made compatible with that of Daubechies (and Pool). To that end we study how the bounded distributions in the space $D_{b}^{\prime}$ studied by Daubechies can be brought into the formalism of this extended product. Happily, they can be fully incorporated into the new product, so that this new formalism extends both that of Antonets and that of Daubechies. Thus armed, we are able to consider the details of the claims of Hansen's proposals.

Since the methods for defining these various products are quite individual, care must be taken when considering combinations of the definitions. In this paper we shall exhibit an approach which provides a unified way of defining a partial algebra structure on the space of tempered distributions, consistent with both of the above definitions. Therefore our single approach can deal with the delicate issues of combining the two approaches, and investigating the properties of the operators of interest. We shall then use our formalism to discover to what extent a spectral calculus can be pushed through. What we achieve is a partial answer to that question, and it is still a moot point as to how adapted the Weyl correspondence is to operator spectral theory.

\section{$\S 2 . \quad$ Basics}

In this section we shall establish the working definitions for the Moyal product on which the rest of our calculations are based. Mainly these are the ones set out in Hansen [9], but with some simplification of notation and of various calculations. The greatest change is that we will work with a onedimensional quantum configuration space, so that $d=1$. Hence phase space is $E=\mathbb{R}^{2}$ (Hansen's choice of notation).

There are two principal references cited in this paper. The first is Hansen's original paper, citation [9], and the second is to the book Mathematical Aspects of Weyl Quantization and Phase by the present authors and T. B. Smith, [5]. 
To save writing, Hansen's paper will sometimes be referred to simply as $[\mathrm{H}]$ and our book as [DHS].

While Hansen's paper involves a number of technical complications, this paper requires considerably more. For example, we introduce several Moyaltype products, including different left and right products thereby denying associativity, several resolvent candidates, none of them analytic in the resolvent, and so on. In point of fact, it is the necessity of these that impelled this work.

Now for the most part, Hansen works with an abstract form of the Moyal product and what is the analogue of the special Hermite functions, and it was tempting to revert to the usual functions and mappings. But we have resisted this temptation as the abstract form subsumes the usual formulation, and by remaining with Hansen's setup we guarantee that our analysis is necessary no matter which formulation is used. To enable the reader to make contact with the usual formulation we have provided an appendix which is mainly a translation dictionary. There we have listed the symbols in [DHS] next to their equivalents in Hansen's notation. We have also shown how Hansen's abstract form of the special Hermite functions maps to the usual realization as functions on $\mathbb{R}^{2}$. We have then used these connections to compare certain expressions that appear here with their [DHS] counterparts.

One question has been avoided so far: is there any advantage in using Hansen's formulation other than making direct contact with his results? Perhaps not, as any other representation of the structures will be equivalent to the usual one. But since Hansen has pioneered this work on a distribution-based approach to spectral theory, it seems best to us to keep to his formulation. The appendix will then provide a path through [DHS] to satisfy those who want to see Laguerre and Hermite functions explicitly, [6, 25].

Thus we shall let $D=\mathcal{S}(E), H=L^{2}(E, d \nu)$, where $(\mid)_{2}$ is the inner product and $\|\cdot\|_{2}$ the norm in $H$, and assume the existence of a jointly continuous associative bilinear map $\star: H \times H \rightarrow H$ such that

$$
\|\xi \star \eta\|_{2} \leq \frac{1}{\sqrt{2}}\|\xi\|_{2} \cdot\|\eta\|_{2}, \quad \overline{\xi \star \eta}=\bar{\eta} \star \bar{\xi}, \quad \xi, \eta \in H .
$$

The measure $\nu$ on $E$ has been normalized, being equal to $\frac{1}{2 \pi}$ times the Lebesgue measure. For more information concerning the measure and the exact nature of the product $\star$ see the Appendix.

The use of lower case Greek letters for vectors in $H$ will be adhered to generally, as will the use of lower case Roman letters for 'test functions' in $D$. Tempered distributions in $D^{\prime}$ will be denoted by the upper case Roman letters $S, T, R, \ldots$, and operators by the other upper case Roman letters identified 
as such at their introduction. The pairing between a distribution $R$ and a test function $f$ will be written $\llbracket R, f \rrbracket$.

The $\star$-product has the property that $D$ is stable under it, and when restricted to $D$ is jointly continuous. The set $\{f \star g: f, g \in D\}$ spans $D$, and the important integral identity

$$
\iint_{E} \xi \star \eta=\iint_{E} \xi \cdot \eta, \quad \xi, \eta \in H
$$

holds. Here $\xi \cdot \eta$ indicates the pointwise product.

Many properties of phase space quantization depend on the projective properties of the Gaussian. Our abstract version begins by positing the existence of an element $\Omega=\bar{\Omega} \in D$ such that

$$
\|\Omega\|_{2}=\sqrt{2},
$$

with projection properties

$$
\Omega \star \Omega=\Omega, \quad \Omega \star \xi \star \Omega=\frac{1}{2}(\Omega \mid \xi)_{2} \Omega, \quad \xi \in H,
$$

relative to the $\star$-product. Then

$$
H_{\Omega}=\{\xi \in H: \xi \star \Omega=\xi\}=\{\xi \star \Omega: \xi \in H\}
$$

is a closed linear subspace of the Hilbert space $H$, while

$$
D_{\Omega}=\{f \in D: f \star \Omega=f\}=\{f \star \Omega: f \in D\}
$$

is a closed linear subspace of the Fréchet space $D$. Moreover $D_{\Omega}$ is dense in $H_{\Omega}$.

The following lemma is a key result showing the interplay between the $\star$ product and the inner product.

Lemma 2.1. If $f, g, u, v \in H_{\Omega}$, then

$$
(f \star \bar{g} \mid u \star \bar{v})_{2}=\frac{1}{2}(f \mid u)_{2}(v \mid g)_{2} .
$$

Proof.

$$
\begin{aligned}
(f \star \bar{g} \mid u \star \bar{v})_{2} & =\iint_{E}(g \star \bar{f}) \cdot(u \star \bar{v})=\iint_{E}(g \star \bar{f}) \star(u \star \bar{v}) \\
& =\iint_{E} g \star(\bar{f} \star u) \star \bar{v}=\frac{1}{2}(f \mid u)_{2} \iint_{E} g \star \Omega \star \bar{v} \\
& =\frac{1}{2}(f \mid u)_{2} \iint_{E} g \star \bar{v}=\frac{1}{2}(f \mid u)_{2} \iint_{E} g \cdot \bar{v} \\
& =\frac{1}{2}(f \mid u)_{2}(v \mid g)_{2}
\end{aligned}
$$


completing the calculation.

The abstract form of the special Hermite functions is based on the existence of a sequence $\left\{a_{n}: n \geq 0\right\} \subseteq D_{\Omega}$ such that:

1. $a_{0}=\Omega$,

2. $\left\{\frac{1}{\sqrt{2}} a_{n}: n \geq 0\right\}$ is an orthonormal basis for $H_{\Omega}$,

3. $\left\{a_{n}: n \geq 0\right\}$ is a Schauder basis for $D_{\Omega}$,

4. the map from the sequence space $\mathfrak{s}$ of rapidly decreasing sequences to $D_{\Omega}$ given by

$$
\left(x_{n}\right)_{n \geq 0} \mapsto \sum_{n \geq 0} x_{n} a_{n}
$$

is a topological linear isomorphism, so the topology on $D_{\Omega}$ may be defined by the family $\left\{p_{k}: k \geq 0\right\}$ of seminorms, where

$$
p_{k}(f)=\sum_{n \geq 0}(n+1)^{k}\left|x_{n}\right|, \quad f=\sum_{n \geq 0} x_{n} a_{n}
$$

For any $m, n \geq 0$, we define the element $a_{m n}=a_{m} \star \overline{a_{n}} \in D$. Then

1. $a_{m 0}=a_{m}$ for all $m \geq 0$,

2. $\overline{a_{m n}}=a_{n m}$ for all $m, n \geq 0$,

3. $a_{m n} \star a_{p q}=\delta_{n p} a_{m q}$ for all $m, n, p, q \geq 0$,

4. $\left\{\frac{1}{\sqrt{2}} a_{m n}: m, n \geq 0\right\}$ is an orthonormal basis for $H$,

5. $\left\{a_{m n}: m, n \geq 0\right\}$ is a Schauder basis for $D$,

6. if we consider the space $\mathfrak{s}^{(2)}$ of rapidly decreasing double sequences, then the map from $\mathfrak{s}^{(2)}$ to $D$ given by

$$
\left(x_{m n}\right)_{m, n \geq 0} \mapsto \sum_{m, n \geq 0} x_{m n} a_{m n}
$$

is a topological isomorphism, so the topology on $D$ can be defined by the family of seminorms $\left\{p_{k l}: k, l \geq 0\right\}$, where

$$
\text { (2.11) } p_{k l}(f)=\sum_{m, n \geq 0}(m+1)^{k}(n+1)^{l}\left|x_{m n}\right|, \quad f=\sum_{m, n \geq 0} x_{m n} a_{m n}
$$


7. if $f \in D$, then $f \in D_{\Omega}$ if and only if $x_{m n}=0$ whenever $n \geq 1$, and moreover

$$
p_{k}(f)=p_{k l}(f), \quad k, l \geq 0 .
$$

All the results listed above can be found in Hansen, $\S 3$ ibid., and demonstrate how we can find the closed linear subspace $H_{\Omega}$ within $H$ which is essentially isomorphic to the Hilbert space $L^{2}(\mathbb{R})$ and how, with respect to this isomorphism, the subspace $D_{\Omega}$ of $D \cap H_{\Omega}$ is isomorphic to the Schwartz space $\mathcal{S}(\mathbb{R})$.

What are these functions $a_{m, n}$ ? In the Appendix we give a realization of the $a_{m, n}$ as particular functions on phase space. The functions $a_{m, n}$ can be identified with the special Hermite function $\phi_{n, m}$ used in [DHS], and this means that the functions $a_{m}$ are to be identified with the functions $\phi_{0, m}$. In [DHS] it is shown that there is a unitary map $L^{2}(\mathbb{R}) \rightarrow H_{\Omega}$ which maps $\sqrt{2 \pi} h_{m}$ to $\oint_{0, m}$, where $h_{m}$ is the $m^{\text {th }}$ Hermite-Gauss function. Thus the functions $a_{m}$ are disguised versions of the Hermite-Gauss functions.

In this regard we note that the matrix multiplication in item 3 is straightforward in the special Hermite function representation, equation (A.7.d) infra. To prove it in the abstract form used above is more difficult, and requires use of equations (2.2) and (2.4).

\section{$\S 3 . \quad$ Bounded Distributions}

In this section we shall see how bounded linear operators on the Hilbert space $H_{\Omega}$ can be related to certain types of bounded linear operators on the full Hilbert space $H$ (which enjoy certain properties with respect to the Moyal product), and we shall also see how these bounded operators on $H_{\Omega}$ can be related to a certain class of tempered distributions in $D^{\prime}$, the so-called bounded distributions, denoted $D_{b}^{\prime}$. The bounded distributions then have a natural structure of a $C^{*}$-algebra inherited from the $C^{*}$-algebra $\mathcal{B}\left(H_{\Omega}\right)$.

\section{§3.1. Preliminaries}

For any $g \in D$ we define the operator $R_{g} \in \mathcal{B}(H)$ of right $\star$-multiplication by setting

$$
R_{g}(\xi)=\xi \star g, \quad \xi \in H,
$$

noting that $R_{g}(D) \subseteq D$. We can consider the norm-closed subalgebra $\mathcal{B}_{*}(H)$ of $\mathcal{B}(H)$ given by

$$
\mathcal{B}_{*}(H)=\left\{X \in \mathcal{B}(H):\left[X, R_{g}\right]=0 \quad \forall g \in D\right\} .
$$




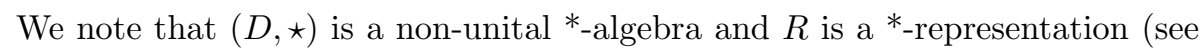
just below for involution). Then $\mathcal{B}_{*}(H)$ is the commutator of $R(D)$.

Lemma 3.1. $\quad\left(R_{g}\right)^{*}=R_{\bar{g}}$ for any $g \in D$.

Using the integral identity (2.2), the proof is a routine calculation.

Proposition 3.1. If $X \in \mathcal{B}_{*}(H)$ then $X\left(H_{\Omega}\right) \subseteq H_{\Omega}$. Moreover the restriction map $J: \mathcal{B}_{*}(H) \rightarrow \mathcal{B}\left(H_{\Omega}\right)$ is an isometric isomorphism of $C^{*}$-algebras.

Proof. Let $X \in \mathcal{B}_{*}(H)$ and $\xi \in H_{\Omega}$. Then $\xi=R_{\Omega} \xi$ and $X \xi=R_{\Omega} X \xi \in$ $H_{\Omega}$. Therefore, $J: \mathcal{B}_{*}(H) \rightarrow \mathcal{B}\left(H_{\Omega}\right)$ is a well-defined linear map, which is clearly norm-decreasing.

We want to construct $K: \mathcal{B}\left(H_{\Omega}\right) \rightarrow \mathcal{B}_{*}(H)$, the linear inverse to $J$. We do this by a limiting process as follows.

Let $Y \in \mathcal{B}\left(H_{\Omega}\right)$ and $\xi=\sum_{m, n \geq 0} \xi_{m n} a_{m n} \in H$. For any $N \geq 0$ define

$$
\xi_{N}=\sum_{m, n=0}^{N} \xi_{m n}\left(Y a_{m}\right) \star \overline{a_{n}}=\sum_{n=0}^{N} Y\left(\sum_{m=0}^{N} \xi_{m n} a_{m}\right) \star \overline{a_{n}} .
$$

We claim that $\left(\xi_{N}\right)$ is a Cauchy sequence in $H$, which we shall prove in detail. Letting $M>N \geq 0$ and using Lemma 2.1 to go from the first to the second line,

$$
\begin{aligned}
& \left\|\xi_{M}-\xi_{N}\right\|_{2}^{2} \\
& \leq\left\|\sum_{n=0}^{N} Y\left(\sum_{m=N+1}^{M} \xi_{m n} a_{m}\right) \star \overline{a_{n}}+\sum_{n=N+1}^{M} Y\left(\sum_{m=0}^{M} \xi_{m n} a_{m}\right) \star \overline{a_{n}}\right\|_{2}^{2} \\
& =\sum_{n=0}^{N}\left\|Y\left(\sum_{m=N+1}^{M} \xi_{m n} a_{m}\right)\right\|_{2}^{2}+\sum_{n=N+1}^{M}\left\|Y\left(\sum_{m=0}^{M} \xi_{m n} a_{m n}\right)\right\|_{2}^{2} \\
& \leq 2\|Y\|^{2}\left(\sum_{n=0}^{N} \sum_{m=N+1}^{M}\left|\xi_{m n}\right|^{2}+\sum_{n=N+1}^{M} \sum_{m=0}^{M}\left|\xi_{m n}\right|^{2}\right) \\
& =2\|Y\|^{2}\left(\sum_{m, n=0}^{M}\left|\xi_{m n}\right|^{2}-\sum_{m, n=0}^{N}\left|\xi_{m n}\right|^{2}\right)
\end{aligned}
$$

so $\lim _{M, N \rightarrow \infty}\left\|\xi_{M}-\xi_{N}\right\|_{2}^{2}=0$. Thus $\left(\xi_{N}\right)_{N \geq 0}$ converges to some element 
$K(Y) \xi \in H$. Since

$$
\begin{aligned}
\left\|\xi_{N}\right\|_{2}^{2} & =\left\|\sum_{n=0}^{N} Y\left(\sum_{m=0}^{N} \xi_{m n} a_{m}\right) \star \overline{a_{n}}\right\|_{2}^{2}=\sum_{n=0}^{N}\left\|Y\left(\sum_{m=0}^{N} \xi_{m n} a_{m}\right)\right\|_{2}^{2} \\
& \leq 2\|Y\|^{2} \sum_{m, n=0}^{N}\left|\xi_{m n}\right|^{2} \leq\|Y\|^{2}\|\xi\|_{2}^{2}
\end{aligned}
$$

for all $N \geq 0$, we deduce that $\|K(Y) \xi\|_{2} \leq\|Y\|\|\xi\|_{2}$. Summarizing, we have defined a linear map $K(Y) \in \mathcal{B}(H)$ with $\|K(Y)\| \leq\|Y\|$ such that

$$
K(Y) a_{m n}=\left(Y a_{m}\right) \star \overline{a_{n}}, \quad m, n \geq 0 .
$$

To prove that $K$ is the inverse of $J$ we must first show that $K(Y) \in \mathcal{B}_{*}(H)$, and to do that we need $K(Y)(\xi \star \eta)$. Now

$$
\begin{aligned}
K(Y)\left(a_{m n} \star a_{p q}\right) & =\delta_{n p} K(Y) a_{m q}=\delta_{n p}\left(Y a_{m}\right) \star \overline{a_{q}}=\left(Y a_{m} \star \overline{a_{n}}\right) \star\left(a_{p} \star \overline{a_{q}}\right) \\
& =\left(K(Y) a_{m n}\right) \star a_{p q}
\end{aligned}
$$

for any $m, n, p, q \geq 0$, so we deduce that

$$
K(Y)(\xi \star \eta)=(K(Y) \xi) \star \eta, \quad \xi, \eta \in H .
$$

This enables us to take the commutator of $K(Y)$ with $R_{g}$ :

$$
\left[K(Y) R_{g}\right](\xi)=K(Y)(\xi \star g)=(K(Y) \xi) \star g=\left[R_{g} K(Y)\right](\xi),
$$

for $\xi \in H, g \in D$, so that $\left[K(Y), R_{g}\right]=0$ for all $g \in D$. This proves that $K(Y) \in \mathcal{B}_{*}(H)$. Thus $K: \mathcal{B}\left(H_{\Omega}\right) \rightarrow \mathcal{B}_{*}(H)$ is a norm-decreasing linear map.

Next we show that $K$ and $J$ are isometric linear inverses. In one direction, let $X \in \mathcal{B}_{*}(H)$. Then

$$
\begin{aligned}
K(J(X)) a_{m n} & =\left(J(X) a_{m}\right) \star \overline{a_{n}}=\left(X a_{m}\right) \star \overline{a_{n}}=R_{\overline{a_{n}}} X a_{m} \\
& =X R_{\overline{a_{n}}} a_{m}=X\left(a_{m} \star \overline{a_{n}}\right)=X a_{m n}
\end{aligned}
$$

for any $m, n \geq 0$, so that $K(J(X))=X$. Conversely, suppose $Y \in \mathcal{B}\left(H_{\Omega}\right)$. Then

$J(K(Y)) a_{m}=K(Y) a_{m}=K(Y) a_{m 0}=\left(Y a_{m}\right) \star \overline{a_{0}}=\left(Y a_{m}\right) \star \Omega=Y a_{m}$ for $m \geq 0$, so that $J(K(Y))=Y$. Thus $J: \mathcal{B}_{*}(H) \rightarrow \mathcal{B}\left(H_{\Omega}\right)$ is an isometric linear bijection with inverse $K$. 
For the next step in the proof we must prove that $J$ is an algebra homomorphism, and at the same time that $\mathcal{B}_{*}(H)$ is an algebra. So let $X, Y \in \mathcal{B}_{*}(H)$. Then $X Y R_{g}=X R_{g} Y=R_{g} X Y$ for $g \in D$, from which it follows that $X Y \in \mathcal{B}_{*}(H)$ and $J(X Y)=J(X) J(Y)$. Thus, as required, $\mathcal{B}_{*}(H)$ is an algebra and $J$ is an algebra homomorphism.

In fact, $\mathcal{B}_{*}(H)$ is a ${ }^{*}$-algebra and $J$ a ${ }^{*}$-homomorphism. To see this, consider $X \in \mathcal{B}_{*}(H)$. Then

$$
X^{*} R_{g}=X^{*}\left(R_{\bar{g}}\right)^{*}=\left(R_{\bar{g}} X\right)^{*}=\left(X R_{\bar{g}}\right)^{*}=\left(R_{\bar{g}}\right)^{*} X^{*}=R_{g} X^{*}
$$

for all $g \in D$, so that $X^{*} \in \mathcal{B}_{*}(H)$ and

$$
\left(\xi \mid J\left(X^{*}\right) \eta\right)_{2}=\left(\xi \mid X^{*} \eta\right)_{2}=(X \xi \mid \eta)_{2}=(J(X) \xi \mid \eta)_{2}=\left(\xi \mid J(X)^{*} \eta\right)_{2}
$$

for $\xi, \eta \in H_{\Omega}$. Thus $\mathcal{B}_{*}(H)$ is a $C^{*}$-subalgebra of $\mathcal{B}(H)$, and $J: \mathcal{B}_{*}(H) \rightarrow$ $\mathcal{B}\left(H_{\Omega}\right)$ is a $C^{*}$-algebra homomorphism. The isometry of $J$ now completes the proof.

In passing we note that we have the standard embeddings $D \subseteq H \subseteq D^{\prime}$, and that the map $T \mapsto \bar{T}$ on $D^{\prime}$ given by

$$
\llbracket \bar{T}, f \rrbracket=\overline{\llbracket T, \bar{f} \rrbracket}, \quad T \in D^{\prime}, f \in D,
$$

is an involution on $D^{\prime}$ which extends the normal involution of complex conjugation on $H$.

\section{§3.2. Properties of bounded distributions}

As previously stated, there is a class of tempered distributions on $E$ that plays a central rôle in the $\star$-multiplication theory.

Definition 3.1. A distribution $T \in D^{\prime}$ is bounded if the set

$$
\left\{\llbracket T, f \star \bar{g} \rrbracket: f, g \in D,\|f\|_{2},\|g\|_{2} \leq 1\right\}
$$

is a bounded subset of $\mathbb{C}$. If $T$ is a bounded distribution, we define its norm to be

$$
\|T\|=\sup \left\{|\llbracket T, f \star \bar{g} \rrbracket|: f, g \in D,\|f\|_{2},\|g\|_{2} \leq 1\right\} .
$$

Let $D_{b}^{\prime}$ denote the collection of all bounded distributions. 
Lemma 3.2. The pair $\left(D_{b}^{\prime},\|\cdot\|\right)$ is a normed space such that

$$
|\llbracket T, f \star \bar{g} \rrbracket| \leq\|T\| \cdot\|f\|_{2} \cdot\|g\|_{2}, \quad T \in D_{b}^{\prime}, \quad f, g \in D,
$$

and, moreover, $H \subseteq D_{b}^{\prime}$ with

$$
\|\xi\| \leq \frac{1}{\sqrt{2}}\|\xi\|_{2}, \quad \xi \in H
$$

Proof. That $D_{b}^{\prime}$ is a normed space, and that the norm satisfies the indicated inequality, is trivial. If $\xi \in H$, then

$$
|\llbracket \xi, f \star \bar{g} \rrbracket|=\left|(\bar{\xi} \mid f \star \bar{g})_{2}\right| \leq\|\xi\|_{2} \cdot\|f \star \bar{g}\|_{2} \leq \frac{1}{\sqrt{2}}\|\xi\|_{2} \cdot\|f\|_{2} \cdot\|g\|_{2}
$$

for any $f, g \in D$, which implies the remaining result.

The terminology 'bounded distribution' is justified by the fact that $D_{b}^{\prime}$ may be identified as a subset of $\mathcal{B}(H)$. The map $\hat{\pi}$ of the next proposition is central to what follows.

Proposition 3.2. Any bounded distribution $T \in D_{b}^{\prime}$ defines a map $\hat{\pi}(T) \in \mathcal{B}_{*}(H)$ with $\|\hat{\pi}(T)\| \leq\|T\|$ by setting

$$
(g \mid \hat{\pi}(T) f)_{2}=\llbracket T, f \star \bar{g} \rrbracket, \quad f, g \in D
$$

Moreover, $\hat{\pi}: D_{b}^{\prime} \rightarrow \mathcal{B}_{*}(H)$ is norm-decreasing.

Proof. Let $T \in D_{b}^{\prime}$. We need only show that $\hat{\pi}(T)$ commutes with every $R_{g}, g \in D$. Let $f, h \in D$, then

$$
\begin{aligned}
& \left(f \mid \hat{\pi}(T) R_{g} h\right)_{2}=(f \mid \hat{\pi}(T)(h \star g))_{2}=\llbracket T,(h \star g) \star \bar{f} \rrbracket=\llbracket T, h \star(g \star \bar{f}) \rrbracket \\
& =\llbracket T, h \star \overline{(f \star \bar{g})} \rrbracket=(f \star \bar{g} \mid \hat{\pi}(T) h)_{2}=\left(\left(R_{g}\right)^{*} f \mid \hat{\pi}(T) h\right)_{2} \\
& =\left(f \mid R_{g} \hat{\pi}(T) h\right)_{2} \text {. }
\end{aligned}
$$

Thus $\left[\hat{\pi}(T), R_{g}\right]=0$ for all $g \in D$, completing the proof.

Corollary 3.1. The definition

$$
\pi_{0}=J \hat{\pi}
$$

determines a norm-decreasing linear map $\pi_{0}: D_{b}^{\prime} \rightarrow \mathcal{B}\left(H_{\Omega}\right)$. 
See the remark at the end of the appendix for a reference to a proof that $\left.\hat{\pi}\right|_{H}$ of $H$ is unitarily equivalent to Weyl quantization. Granting this, there is no need continually to refer to Weyl quantization in what follows.

The space of bounded distributions and the associated maps $\hat{\pi}$ and $\pi_{0}$, behave well with respect to the involution in $D^{\prime}$.

Lemma 3.3. If $T \in D_{b}^{\prime}$ then $\bar{T} \in D_{b}^{\prime}$ and $\hat{\pi}(\bar{T})=\hat{\pi}(T)^{*}, \pi_{0}(\bar{T})=$ $\pi_{0}(T)^{*}$.

Proof. Let $T \in D_{b}^{\prime}$ and $f, g \in D$. Since

$$
|\llbracket \bar{T}, f \star \bar{g} \rrbracket|=|\llbracket T, g \star \bar{f} \rrbracket| \leq\|T\| \cdot\|f\|_{2} \cdot\|g\|_{2},
$$

it follows that $\bar{T} \in D_{b}^{\prime}$ with $\|\bar{T}\| \leq\|T\|$. To prove equality we note that $\overline{\bar{T}}=T$ implies $\|\bar{T}\|=\|T\|$. From the definition of $\hat{\pi}$,

$$
\begin{aligned}
(f \mid \hat{\pi}(\bar{T}) g)_{2} & =\llbracket \bar{T}, g \star \bar{f} \rrbracket=\overline{\llbracket T, f \star \bar{g} \rrbracket}=\overline{(g \mid \hat{\pi}(T) f)_{2}} \\
& =(\hat{\pi}(T) f \mid g)_{2}=\left(f \mid \hat{\pi}(T)^{*} g\right)_{2},
\end{aligned}
$$

showing that $\hat{\pi}(\bar{T})=\hat{\pi}(T)^{*}$. As $J$ is a *-homomorphism it is immediate that $\pi_{0}(\bar{T})=\pi_{0}(T)^{*}$.

It has now been shown that the bounded distributions may be represented as bounded operators on $H_{\Omega}$. More is true: under the map $\pi_{0}$ they comprise all the bounded operators.

Proposition 3.3. The maps $\hat{\pi}: D_{b}^{\prime} \rightarrow \mathcal{B}_{*}(H)$ and $\pi_{0}: D_{b}^{\prime} \rightarrow \mathcal{B}\left(H_{\Omega}\right)$ are isometric linear bijections.

Proof. We want to construct a norm-decreasing map $L: \mathcal{B}\left(H_{\Omega}\right) \rightarrow D_{b}^{\prime}$ inverse to $\pi_{0}$. To begin, let $Y \in \mathcal{B}\left(H_{\Omega}\right)$. Then

$$
\left|\left(a_{n} \mid Y a_{m}\right)_{2}\right| \leq\left\|Y a_{m}\right\|_{2} \cdot\left\|a_{n}\right\|_{2} \leq 2\|Y\|
$$

for all $m, n \geq 0$, By continuous linear extension we can use this to define the element $L(Y) \in D^{\prime}$ by setting

$$
\llbracket L(Y), f \rrbracket=\sum_{m, n \geq 0} f_{m n}\left(a_{n} \mid Y a_{m}\right)_{2}, \quad f=\sum_{m, n \geq 0} f_{m n} a_{m n} \in D,
$$

with

$$
|\llbracket L(Y), f \rrbracket| \leq 2\|Y\| p_{00}(f), \quad f \in D .
$$


The mapping $L$ can be related to the mapping $K$, the inverse of $J$,

$$
\begin{aligned}
\llbracket L(Y), a_{m n} \star a_{p q} \rrbracket & =\delta_{n p} \llbracket L(Y), a_{m q} \rrbracket=\delta_{n p}\left(a_{q} \mid Y a_{m}\right)_{2} \\
& =\left(a_{q} \mid\left(Y a_{m}\right) \star \overline{a_{n}} \star a_{p}\right)_{2}=\left(a_{q} \mid R_{a_{p}}\left[\left(Y a_{m}\right) \star \overline{a_{n}}\right]\right)_{2} \\
& =\left(R_{\overline{a_{p}}}\left(a_{q}\right) \mid\left(Y a_{m}\right) \star \overline{a_{n}}\right)_{2}=\left(a_{q} \star \overline{a_{p}} \mid K(Y) a_{m n}\right)_{2} \\
& =\left(\overline{a_{p q}} \mid K(Y) a_{m n}\right)_{2}
\end{aligned}
$$

for $m, n, p, q \geq 0$, so that by linearity and continuity,

$$
\llbracket L(Y), f \star \bar{g} \rrbracket=(g \mid K(Y) f)_{2}, \quad f, g \in D .
$$

This result can be used to show that $L(Y)$ is a bounded distribution:

$$
|\llbracket L(Y), f \star \bar{g} \rrbracket| \leq\|K(Y) f\|_{2} \cdot\|g\|_{2} \leq\|K(Y)\| \cdot\|f\|_{2} \cdot\|g\|_{2} \leq\|Y\|
$$

for all $f, g \in D$ with $\|f\|_{2},\|g\|_{2} \leq 1$, implying that $L(Y) \in D_{b}^{\prime}$, with $\|L(Y)\| \leq\|Y\|$. The map $L: \mathcal{B}\left(H_{\Omega}\right) \rightarrow D_{b}^{\prime}$ has now been constructed and shown to be norm-decreasing.

We claim that $\pi_{0}(L(\cdot))$ is the identity map on $\mathcal{B}\left(H_{\Omega}\right)$. Let $Y \in \mathcal{B}\left(H_{\Omega}\right)$. Then

$$
(g \mid \hat{\pi}(L(Y)) f)_{2}=\llbracket L(Y), f \star \bar{g} \rrbracket=(g \mid K(Y) f)_{2}
$$

for all $f, g \in D$, and hence $\hat{\pi}(L(Y))=K(Y)$. This in turn implies that $\pi_{0}(L(Y))=Y$, proving the claim.

Conversely we claim that $L\left(\pi_{0}(\cdot)\right)$ is the identity on $D_{b}^{\prime}$. Let $T \in D_{b}^{\prime}$. Then

$$
\begin{aligned}
\llbracket L\left(\pi_{0}(T)\right), a_{m n} \rrbracket & =\left(a_{n} \mid \pi_{0}(T) a_{m}\right)_{2}=\left(a_{n} \mid \hat{\pi}(T) a_{m}\right)_{2} \\
& =\llbracket T, a_{m} \star \overline{a_{n}} \rrbracket=\llbracket T, a_{m n} \rrbracket
\end{aligned}
$$

for all $m, n \geq 0$, so that $L\left(\pi_{0}(X)\right)=T$, as required.

Hence we deduce that $\pi_{0}: D_{b}^{\prime} \rightarrow \mathcal{B}\left(H_{\Omega}\right)$ is an isometric linear bijection with inverse $L$, and moreover that $\hat{\pi}: D_{b}^{\prime} \rightarrow \mathcal{B}_{*}(H)$ is also an isometric linear bijection with inverse $L \star J$.

Thus we can use the algebraic structure of $\mathcal{B}\left(H_{\Omega}\right)$ to deduce an algebraic structure on $D_{b}^{\prime}$. We can define an associative algebraic product $\star_{b}$ on $D_{b}^{\prime}$ by the formulae:

$$
\begin{aligned}
\hat{\pi}\left(S \star_{b} T\right) & =\hat{\pi}(S) \hat{\pi}(T), & S, T \in D_{b}^{\prime}, \\
\pi_{0}\left(S \star_{b} T\right) & =\pi_{0}(S) \pi_{0}(T), & S, T \in D_{b}^{\prime} .
\end{aligned}
$$

With this product and complex conjugation as involution, $\left(D_{b}^{\prime},\|\cdot\|, \star_{b}, \overline{ }\right)$ becomes a $C^{*}$-algebra, and the three maps $\hat{\pi}: D_{b}^{\prime} \rightarrow \mathcal{B}_{*}(H), J: \mathcal{B}_{*}(H) \rightarrow$ 
$\mathcal{B}\left(H_{\Omega}\right)$, and $\pi_{0}: D_{b}^{\prime} \rightarrow \mathcal{B}\left(H_{\Omega}\right)$, are $C^{*}$-algebra isomorphisms such that $\pi_{0}=$ $J \hat{\pi}$. Thus the bounded distributions $D_{b}^{\prime}$ correspond precisely with the bounded multipliers on $H$, and with the bounded operators on $H_{\Omega}$. An obvious first task is to identify some of the elements of $D_{b}^{\prime}$. We have already seen that $H \subseteq D_{b}^{\prime}$, and elementary calculations show that

$$
\hat{\pi}(\xi) \eta=\xi \star \eta, \quad \xi, \eta \in H .
$$

However, the properties of the operators corresponding to elements of $H$ can be more precisely specified than this. The result is fairly straightforward and, in view of the results of Pool, [20], not surprising.

Proposition 3.4. $\quad \pi_{0}(H)=\mathcal{H} S\left(H_{\Omega}\right)$ is the set of Hilbert-Schmidt operators on $H_{\Omega}$, with

$$
\left\|\pi_{0}(\xi)\right\|_{\mathcal{H S S}}=\frac{1}{\sqrt{2}}\|\xi\|_{2}, \quad \xi \in H .
$$

Proof. We begin by showing that $\pi_{0}(\xi)$ is left $\star$-multiplication by $\xi$ on $H_{\Omega}$ for all $\xi \in H$. Since $H_{\Omega}$ is a proper subset of $H$, this action of $\pi_{0}$ is not an extension of $R_{g}$ with $g \in D$, strictly speaking.

Let $\xi \in H$ and $f, g \in D_{\Omega}$. Then

$$
\left(f \mid \pi_{0}(\xi) g\right)_{2}=\llbracket \xi, g \star \bar{f} \rrbracket=\iint_{E} \xi \cdot(g \star \bar{f})=\iint_{E}(\xi \star g) \cdot \bar{f}=(f \mid \xi \star g)_{2},
$$

so that $\pi_{0}(\xi) g=\xi \star g$ for $g \in D_{\Omega}$, and so by continuous extension, $\pi_{0}(\xi) \eta=$ $\xi \star \eta$ for $\eta \in H_{\Omega}$.

With this characterization we can now show the Hilbert-Schmidt property. If $\xi=\sum_{m, n \geq 0} \xi_{m n} a_{m n} \in H$, then

$$
\pi_{0}(\xi) a_{q}=\xi \star a_{q}=\sum_{m \geq 0} \xi_{m q} a_{m} .
$$

Taking norms,

$$
\left\|\pi_{0}(\xi) a_{q}\right\|_{2}^{2}=\left\|\sum_{m \geq 0} \xi_{m q} a_{m}\right\|_{2}^{2}=2 \sum_{m \geq 0}\left|\xi_{m q}\right|^{2}
$$

and then summing over $q$,

$$
\sum_{q \geq 0}\left\|\pi_{0}(\xi) a_{q}\right\|_{2}^{2}=2 \sum_{m, q \geq 0}\left|\xi_{m q}\right|^{2}=\|\xi\|_{2}^{2}
$$


Since $\left\{a_{q} / \sqrt{2}: q \geq 0\right\}$ is an orthonormal basis for $H_{\Omega}$, it follows that $\pi_{0}(\xi)$ is a Hilbert-Schmidt operator on $H_{\Omega}$, with $2\left\|\pi_{0}(\xi)\right\|_{\mathcal{H} S}^{2}=\|\xi\|_{2}^{2}$, as required.

On the other hand, if $T \in D_{b}^{\prime}$ is such that $\pi_{0}(T) \in \mathcal{H} \&\left(H_{\Omega}\right)$, then

$$
\begin{aligned}
\frac{1}{4} \sum_{m, n \geq 0}\left|\llbracket T, a_{m n} \rrbracket\right|^{2} & =\frac{1}{4} \sum_{m, n \geq 0}\left|\left(a_{n} \mid \pi_{0}(T) a_{m}\right)_{2}\right|^{2}=\frac{1}{2} \sum_{m \geq 0}\left\|\pi_{0}(T) a_{m}\right\|_{2}^{2} \\
& =\left\|\pi_{0}(T)\right\|_{\mathcal{H} S}^{2},
\end{aligned}
$$

so the two-index sequence $\left(\llbracket T, a_{m n} \rrbracket\right)_{m, n \geq 0}$ belongs to $\ell_{2}^{2}$. Thus we can define $\xi \in H$ by setting

$$
\xi=\frac{1}{2} \sum_{m, n \geq 0} \llbracket T, a_{n m} \rrbracket a_{m n},
$$

observing that

$$
\|\xi\|_{2}^{2}=\frac{1}{2} \sum_{m, n \geq 0}\left|\llbracket T, a_{m n} \rrbracket\right|^{2}=2\left\|\pi_{0}(T)\right\|_{\mathcal{H} \mathcal{S}}^{2} .
$$

Now since

$$
\xi\left(a_{p q}\right)=\left(\overline{a_{p q}} \mid \xi\right)_{2}=\left(a_{q p} \mid \xi\right)_{2}=\llbracket T, a_{p q} \rrbracket
$$

for all $p, q \geq 0$, it is clear that $T=\xi \in H$.

The similarity to the expansion by means of a resolution of the identity in the definition of $\xi$ will not be lost on the reader.

Thus the bounded distributions can be characterized as those elements of $D^{\prime}$ which correspond to bounded multipliers of $H$, or equivalently to bounded operators on $H_{\Omega}$, while elements of $H$, considered as elements of $D_{b}^{\prime}$, correspond to Hilbert-Schmidt operators on $H_{\Omega}$. For $\xi \in H \subseteq D^{\prime}$ we see that

$$
\begin{aligned}
\hat{\pi}(\xi) \eta & =\xi \star \eta, & & \eta \in H, \\
\pi_{0}(\xi) \eta & =\xi \star \eta, & & \eta \in H_{\Omega},
\end{aligned}
$$

and, while the restrictions of $\hat{\pi}(\xi)$ to $D$ and of $\pi_{0}(\xi)$ to $D_{\Omega}$ are endomorphisms of $D$ and of $D_{\Omega}$ respectively when $\xi \in D$, these identifications are not true when $\xi$ is a general element of $H$. Thus the algebra structure of $D_{b}^{\prime}$ is obtained by looking from the operators acting on the whole Hilbert space $H$ (or $H_{\Omega}$ ), and not by acting on the Fréchet subspaces $D$ and $D_{\Omega}$. In the next section, we shall consider classes of distributions for which partial algebra structures based upon on the Moyal product can be defined through concentrating on these Fréchet subspaces. 


\section{$\S 4 . \quad$ Extending the Product to Tempered Distributions}

In this section, we shall start the process of extending the Moyal product so that it can be applied to tempered distributions, and not just functions. One way of doing this is as follows: given a function $g \in D$, the right-multiplication map $R_{g}: D \rightarrow D$ can be extended to yield a map $T \mapsto T \star g$ from $D^{\prime}$ to itself. Similarly, the analogously defined left-multiplication map $L_{g}$ on $D$ can be extended to yield a map $T \mapsto g \star T$ from $D^{\prime}$ to itself. Although these two constructions are similar, there are enough subtle distinctions between them that it is easy to make mistakes if the two are not distinguished, so we are forced to introduce different notations for the two products.

This is complicated enough, but we want more. In particular, we want to be able to extend $\star$ in some way so that the product of two distributions is possible, not just the product of a distribution and a function. It will not be possible to define such a product on all distributions, so a judgment has to be made about the choice of distributions on which to define this extended product. In this section we consider a number of possible spaces of distributions. The first two, which we have denoted $N$ and $\bar{N}$, are such that the extended product turns them into associative algebras. Unfortunately, they suffer from certain defects. While they are both large sets containing $D$ they have a large overlap with $D_{b}^{\prime}$ but do not contain it, [7]. Nor even do they contain $H$. Thus while Hansen has proved an associativity result for his Moyal product (his equation (5.16)) valid for the triple $\bar{N} \times D_{b}^{\prime} \times H$, the fact that this result only holds when the three elements belong to very particular spaces, $\bar{N}, H$, and $D_{b}^{\prime}$, makes this result of limited utility, since the circumstances under which it can be used are comparatively limited, compared with what might be desired.

The second two spaces, which we have called $Q$ and $\bar{Q}$, both contain $D_{b}^{\prime}$. It is not possible to extend $\star$ to either of them, but we can define the product of an element of $\bar{Q}$ with an element of $Q$. Thus we can define a product on $Q \cap \bar{Q}$. However, this product is not associative, so we do not have an algebra.

Thus there are two natural ways of extending the original Moyal product $\star ~: D \times D \rightarrow D$ to give bilinear maps, $D \times D^{\prime} \rightarrow D^{\prime}$ and $D^{\prime} \times D \rightarrow D^{\prime}$, and each of these can be used to obtain a partial algebra structure [1] on $D^{\prime}$. After defining the two products, our first aim is to see to what extent they agree with each other.

The results in this section are not new - except perhaps for the results relating the two products (see for example Kammerer [12]). Although the proofs are not difficult, they are included for the purposes of completeness. 
Definition 4.1. Since $\star: D \times D \rightarrow D$ is a continuous bilinear map, we can define bilinear maps $\star_{1}: D^{\prime} \times D \rightarrow D^{\prime}$ and $\star_{2}: D \times D^{\prime} \rightarrow D^{\prime}$ by setting

$$
\begin{aligned}
& \llbracket T \star_{1} f, g \rrbracket=\llbracket T, f \star g \rrbracket \quad T \in D^{\prime}, \quad f, g \in D, \\
& \llbracket f \star_{2} T, g \rrbracket=\llbracket T, g \star f \rrbracket \quad T \in D^{\prime}, \quad f, g \in D .
\end{aligned}
$$

Previously proved properties of $\star$ allows us to obtain some elementary properties of these new maps.

Lemma 4.1. The maps $\star_{1}$ and $\star_{2}$ possess the following properties: for all $f, g \in D, \xi \in H$, and $T \in D^{\prime}$,

1. $\xi \star_{1} g=\xi \star g, \quad g \star_{2} \xi=g \star \xi ;$

2. $\left(T \star_{1} f\right) \star_{1} g=T \star_{1}(f \star g)$;

3. $f \star_{2}\left(g \star_{2} T\right)=(f \star g) \star_{2} T$;

4. $\overline{T \star_{1} f}=\bar{f} \star_{2} \bar{T}$;

5. $f \star_{2}\left(T \star_{1} g\right)=\left(f \star_{2} T\right) \star_{1} g$;

6. $1 \star_{1} f=f \star_{2} 1=f$, where $1 \in D^{\prime}$ is the tempered distribution

$$
\llbracket 1, f \rrbracket=\iint_{E} f .
$$

Proof. The first result follows since

$$
\begin{aligned}
\llbracket \xi \star_{1} g, f \rrbracket & =\llbracket \xi, g \star f \rrbracket=\iint_{E} \xi \cdot(g \star f)=\iint_{E}(\xi \star g) \cdot f=\llbracket \xi \star g, f \rrbracket, \\
\llbracket g \star_{2} \xi, f \rrbracket & =\llbracket \xi, f \star g \rrbracket=\iint_{E} \xi \cdot(f \star g)=\iint_{E}(\xi \star f) \cdot g \\
& =\iint_{E} g \cdot(\xi \star f)=\iint_{E}(g \star \xi) \cdot f=\llbracket g \star \xi, f \rrbracket,
\end{aligned}
$$

for $f \in D$. The second follows since

$$
\begin{aligned}
\llbracket\left(T \star_{1} f\right) \star_{1} g, h \rrbracket & =\llbracket T \star_{1} f, g \star h \rrbracket=\llbracket T, f \star(g \star h) \rrbracket \\
& =\llbracket T,(f \star g) \star h \rrbracket=\llbracket T \star_{1}(f \star g), h \rrbracket
\end{aligned}
$$

for $h \in D$, while the fourth is true since

$$
\begin{aligned}
\overline{\llbracket T \star_{1} f, g} \rrbracket & =\overline{\llbracket T \star_{1} f, \bar{g} \rrbracket}=\overline{\llbracket T,(f \star \bar{g}) \rrbracket} \\
& =\llbracket \bar{T}, g \star \bar{f} \rrbracket=\llbracket \bar{f} \star_{2} \bar{T}, g \rrbracket
\end{aligned}
$$


for $g \in D$. The third result is then an immediate consequence of the second and the fourth. Since

$\llbracket f \star_{2}\left(T \star_{1} g\right), h \rrbracket=\llbracket T, g \star(h \star f) \rrbracket=\llbracket T,(g \star h) \star f \rrbracket=\llbracket\left(f \star_{2} T\right) \star_{1} g, h \rrbracket$

for $h \in D$, we deduce the fifth result, and the last follows since

$$
\llbracket 1 \star_{1} f, g \rrbracket=\iint_{E} f \star g=\llbracket f, g \rrbracket=\iint_{E} g \star f=\llbracket f \star_{2} 1, g \rrbracket
$$

for all $f, g \in D$.

The particular classes of distributions mentioned at the beginning of this section are specified by restricting attention to those distributions $T \in D^{\prime}$ whose products with elements of $D$ are functions, and not more general distributions. More specifically, we look at distributions whose products with elements of $D$ either belong to $D$ or to $H$. Thus we are led to the following subspaces of $D^{\prime}$ :

$$
\begin{array}{ll}
N=\left\{T \in D^{\prime}: T \star_{1} f \in D\right. & \forall f \in D\}, \\
\bar{N}=\left\{T \in D^{\prime}: f \star_{2} T \in D\right. & \forall f \in D\}, \\
Q=\left\{T \in D^{\prime}: T \star_{1} f \in H\right. & \forall f \in D\}, \\
\bar{Q}=\left\{T \in D^{\prime}: f \star_{2} T \in H\right. & \forall f \in D\} .
\end{array}
$$

The first two spaces are, essentially, those used by Hansen [9], but the second two are new, and closely resemble the space of quasi-observables discussed in DHS [5]. Out first task is to investigate the set-theoretic relations between these four spaces and the space $D_{b}^{\prime}$ of bounded observables. The first few results are elementary.

Lemma 4.2. The following results can be established:

1. $T \in N$ if and only if $\bar{T} \in \bar{N}$,

2. $T \in Q$ if and only if $\bar{T} \in \bar{Q}$,

3. if $T \in D_{b}^{\prime}$ then

$$
\begin{aligned}
& \text { (4.4.a) } T \star_{1} f=\hat{\pi}(T) f, \bar{f} \star_{2} T=\overline{\hat{\pi}(\bar{T}) f}, \quad f \in D \text {, } \\
& \text { (4.4.b) } T \star_{1} f=\pi_{0}(T) f, \bar{f} \star_{2} T=\overline{\pi_{0}(\bar{T}) f}, \quad f \in D_{\Omega} \text {. }
\end{aligned}
$$

4. $D_{b}^{\prime}+N \subseteq Q$. 
Proof. Since $\bar{f} \star_{2} \bar{T}=\overline{T \star_{1} f}$ for any $T \in D^{\prime}$ and $f \in D$, the first and second results are evident. If $T \in D_{b}^{\prime}$ then

$$
\llbracket T \star_{1} f, g \rrbracket=\llbracket T, f \star g \rrbracket=(\bar{g} \mid \hat{\pi}(T) f)_{2}=\llbracket \hat{\pi}(T) f, g \rrbracket
$$

for $f, g \in D$, so that $T \star_{1} f=\hat{\pi}(T) f$ for $f \in D$. The formula for $\bar{f} \star_{2} T$ is obtained from this first by conjugation. The formulae for $f \in D_{\Omega}$ simply follow from these two by restriction. $Q$.

It is clear that $D_{b}^{\prime} \subseteq Q \cap \bar{Q}$, and that $N \subseteq Q$, and so certainly $D_{b}^{\prime}+N \subseteq$

Corollary 4.1. For any $T \in Q$, we define a map $\pi(T) \in \operatorname{Hom}(D, H)$ by setting

$$
\pi(T) f=T \star_{1} f, \quad f \in D,
$$

noting that $\pi(T) \in \operatorname{End}(D)$ if $T \in N$. Moreover, the restriction of $\pi(T)$ to $D_{\Omega}$ is a homomorphism from $D_{\Omega}$ to $H_{\Omega}$ for $T \in Q$, and an endomorphism of $D_{\Omega}$ when $T \in N$.

Proof. Comparing the definition of $\pi(T)$ with (4.3.a) and (4.3.c) shows it to be a homomorphism from $D$ to $H$ if $T \in Q$ and from $D$ to $D$ if $T \in N$.

The results about the restriction of $\pi(T)$ follow from the fact that $\pi(T)$ acts on the left in a $\star$-pair:

$$
\pi(T)(f \star g)=T \star_{1}(f \star g)=\left(T \star_{1} f\right) \star_{1} g=\left(T \star_{1} f\right) \star g=(\pi(T) f) \star g
$$

for all $f, g \in D$. Thus, if $f \in D_{\Omega}$, we have that

$$
\pi(T)(f)=T \star_{1}(f \star \Omega)=(\pi(T) f) \star \Omega
$$

belongs to $H_{\Omega}$. That is, $\pi(T)$ maps $D_{\Omega}$ into $H_{\Omega}$. A similar argument yields the result for $T \in N$.

So far we have only considered the algebraic structure of these maps, but it can be shown that all the maps defined above are continuous when $D$ carries its Fréchet topology. In addition, it is also possible to begin to see the connection between the two definitions of an algebra structure for the bounded distributions.

Lemma 4.3. As usual, we write $\mathcal{L}(E, F)$ for the set of continuous linear maps from $E$ to $F$, where $E$ and $F$ are topological linear spaces, and $\mathcal{L}(E, E)=\mathcal{L}(E)$. With this notation, the following results can be obtained: 
1. If $T \in Q$ then $\pi(T) \in \mathcal{L}(D, H)$ and its restriction to $D_{\Omega}$ belongs to $\mathcal{L}\left(D_{\Omega}, H_{\Omega}\right)$

2. If $T \in N$ then $\pi(T) \in \mathcal{L}(D)$ and its restriction to $D_{\Omega}$ belongs to $\mathcal{L}\left(D_{\Omega}\right)$,

3. If $T \in D_{b}^{\prime}$ then $\pi(T) \leq \hat{\pi}(T)$ and $\left.\pi(T)\right|_{D_{\Omega}} \leq \pi_{0}(T)$, where $\leq$ indicates extension.

Proof.

1. Let $T \in Q$. We will use the closed graph theorem to prove this result, so suppose that $f \in D,\left(f_{n}\right)_{n} \subseteq D$, and $\xi \in H$ are such that $f_{n} \rightarrow f$ in $D$ and $\pi(T) f_{n} \rightarrow \xi$ in $H$. Then

$$
\begin{aligned}
(h \mid \pi(T) f)_{2} & =\llbracket T \star_{1} f, \bar{h} \rrbracket=\llbracket T, f \star \bar{h} \rrbracket=\lim _{n \rightarrow \infty} \llbracket T, f_{n} \star \bar{h} \rrbracket \\
& =\lim _{n \rightarrow \infty} \llbracket T \star_{1} f_{n}, \bar{h} \rrbracket=\lim _{n \rightarrow \infty}\left(h \mid \pi(T) f_{n}\right)_{2}=(h \mid \xi)_{2}
\end{aligned}
$$

for all $h \in D$, so that $\pi(T) f=\xi$. Thus $\pi(T) \in \operatorname{Hom}(D, H)$ has a closed graph, and so is continuous. We conclude that $\pi(T) \in \mathcal{L}(D, H)$, and the result about its restriction is now automatic.

2. Now let $T \in N$, and suppose that $f, g \in D$ and $\left(f_{n}\right)_{n} \subseteq D$ are such that $f_{n} \rightarrow f$ in $D$ and $\pi(T) f_{n} \rightarrow g$ in $D$. Then

$$
\begin{aligned}
(h \mid \pi(T) f)_{2} & =\llbracket T \star_{1} f, \bar{h} \rrbracket=\llbracket T, f \star \bar{h} \rrbracket=\lim _{n \rightarrow \infty} \llbracket T, f_{n} \star \bar{h} \rrbracket \\
& =\lim _{n \rightarrow \infty}\left(h \mid \pi(T) f_{n}\right)_{2}=(h \mid g)_{2}
\end{aligned}
$$

for all $h \in D$, so that $\pi(T) f=g$. Using the Closed Graph Theorem again, we deduce that $\pi(T) \in \mathcal{L}(D)$, which implies the result about the restriction.

3. Now suppose that $T \in D_{b}^{\prime}$. Since $\pi(T) f=T \star_{1} f=\hat{\pi}(T) f$ for all $f \in D$, we see that $\pi(T) \leq \hat{\pi}(T)$. Since $\pi_{0}(T)=\left.\hat{\pi}(T)\right|_{H_{\Omega}}$, we have $\left.\pi(T)\right|_{D_{\Omega}} \leq \pi_{0}(T)$ as well.

There is no particular need to distinguish between a map $\pi(T)$ and its restriction to $D_{\Omega}$, and hence we shall use the symbol $\pi(T)$ to refer to either map. Context should make clear which map we mean at any particular time. 


\section{$\S 5 . \quad$ The Moyal Product of Two Distributions}

In this section we shall investigate a new $\star$-product, $\hat{\star}$, one which allows us to take the Moyal product of many (but not all) pairs of distributions in $D^{\prime}$. To date we have considered a variety of products - $-\star_{b}, \star_{1}, \star_{2}-$ defined on a variety of spaces - D, H, D $, N, \bar{N}, Q, \bar{Q}$ — and this new product $\hat{\star}$ attempts to bring all of these definitions together under a single umbrella (so far as this is possible). We shall determine the relationship between this product and the more obvious products that already exist between two bounded distributions or between two elements of $N$ as discussed in [9].

Proposition 5.1. The equation

$$
\llbracket S \star T, f \star \bar{g} \rrbracket=\left(\bar{S} \star_{1} g \mid T \star_{1} f\right)_{2}, \quad f, g \in D .
$$

determines a bilinear mapping $\hat{\star}: \bar{Q} \times Q \rightarrow D^{\prime}$.

Proof. If $T \in Q$, then $\pi(T) \in \mathcal{L}\left(D_{\Omega}, H_{\Omega}\right)$, and so we can find $A>0$ and $k \geq 0$ such that

$$
\|\pi(T) f\|_{2} \leq A p_{k}(f) \quad f \in D_{\Omega},
$$

and hence

$$
\left\|T \star_{1} a_{n}\right\|_{2}=\left\|\pi(T) a_{n}\right\|_{2} \leq A p_{k}\left(a_{n}\right)=A(n+1)^{k}, \quad n \geq 0 .
$$

Thus, if $S \in \bar{Q}$ and $T \in Q$, we can find $A, B>0$ and $k, l \geq 0$ such that

$$
\left\|\bar{S} \star_{1} a_{n}\right\|_{2} \leq A(n+1)^{k} \quad\left\|T \star_{1} a_{n}\right\|_{2} \leq B(n+1)^{l}, \quad n \geq 0,
$$

which implies that

$$
\left|\left(\bar{S} \star_{1} a_{n} \mid T \star_{1} a_{m}\right)_{2}\right| \leq A B(m+1)^{l}(n+1)^{k}, \quad m, n \geq 0 .
$$

Thus we can define a distribution $S \hat{\star} T \in D^{\prime}$ such that

$$
\llbracket S \hat{\star} T, a_{m n} \rrbracket=\left(\bar{S} \star_{1} a_{n} \mid T \star_{1} a_{m}\right)_{2}, \quad m, n \geq 0,
$$

noting that $|\llbracket S \hat{\star} T, f \rrbracket| \leq A B p_{l k}(f)$ for any $f \in D$. Now

$$
\begin{aligned}
\llbracket S \hat{\star} T, a_{m n} \star \overline{a_{p q}} \rrbracket & =\llbracket S \hat{\star} T, a_{m n} \star a_{q p} \rrbracket=\delta_{n q} \llbracket S \hat{\star} T, a_{m p} \rrbracket \\
& =\delta_{n q}\left(\bar{S} \star_{1} a_{p} \mid T \star_{1} a_{m}\right)_{2}=\left(\bar{S} \star_{1} a_{p} \mid\left(T \star_{1} a_{m}\right) \star \overline{a_{n}} \star a_{q}\right)_{2} \\
& \left.=\left(\bar{S} \star_{1} a_{p}\right) \star \overline{a_{q}} \mid\left(T \star_{1} a_{m}\right) \star \overline{a_{n}}\right)_{2} \\
& =\left(\bar{S} \star_{1}\left(a_{p} \star \overline{a_{q}}\right) \mid T \star_{1}\left(a_{m} \star \overline{a_{n}}\right)\right)_{2} \\
& =\left(\bar{S} \star_{1} a_{p q} \mid T \star_{1} a_{m n}\right)_{2}
\end{aligned}
$$


for all $m, n, p, q \geq 0$, from which we deduce that

$$
\llbracket S \hat{\star} T, f \star \bar{g} \rrbracket=\left(\bar{S} \star_{1} g \mid T \star_{1} f\right)_{2}, \quad f, g \in D .
$$

Complex conjugation maps $Q$ to $\bar{Q}$ and conversely, so we should investigate the behaviour of $\hat{\star}$ under complex conjugation,

Lemma 5.1. If $S \in \bar{Q}$ and $T \in Q$, then $\overline{S \hat{\star} T}=\bar{T} \hat{\star} \bar{S}$.

Proof. For all $f, g \in D$,

$$
\begin{aligned}
\llbracket \overline{S \hat{\star} T}, f \star \bar{g} \rrbracket & =\overline{(S \hat{\star} T)(g \star \bar{f})}=\overline{\left(\bar{S} \star_{1} f \mid T \star_{1} g\right)_{2}} \\
& =\left(T \star_{1} g \mid \bar{S} \star_{1} f\right)_{2}=\llbracket \bar{T} \hat{S}, \bar{f} \star g \rrbracket,
\end{aligned}
$$

which establishes the result.

The next lemma shows that $\hat{\star}$ is indeed a product which extends the previous products that we have considered:

Lemma 5.2. The following statements are true:

1. If $S, T \in D_{b}^{\prime}$, then $S \hat{\star} T=S \star_{b} T$,

2. If $\xi, \eta \in H$ then $\xi \hat{\star} \eta=\xi \star \eta$,

3. If $T \in \bar{Q}$ and $f \in D$ then $T \hat{\star} f=T \star_{1} f$,

4. If $T \in Q$ and $f \in D$ then $f \hat{\star} T=f \star_{2} T$,

5. If $S \in \bar{Q}$ and $T \in N$ then $\llbracket S \hat{\star} T, f \rrbracket=\llbracket S, T \star_{1} f \rrbracket$ for all $f \in D$,

6. If $S, T \in N \cap \bar{N}$ then $S \hat{\star} T \in N \cap \bar{N}$.

Proof.

1. Since

$$
\begin{aligned}
\llbracket S \hat{\star} T, f \star \bar{g} \rrbracket & =(\hat{\pi}(\bar{S}) g \mid \hat{\pi}(T) f)_{2}=(g \mid \hat{\pi}(S) \hat{\pi}(T) f)_{2} \\
& =\left(g \mid \hat{\pi}\left(S \star_{b} T\right) f\right)_{2}
\end{aligned}
$$

for all $f, g \in D$, we see that $S \hat{\star} T=S \star_{b} T$. 
2. We see that

$$
\hat{\pi}(\xi \hat{\star} \eta) g=\hat{\pi}\left(\xi \star_{b} \eta\right) g=\hat{\pi}(\xi) \hat{\pi}(\eta) g=\xi \star(\eta \star g)=\hat{\pi}(\xi \star \eta) g
$$

for all $g \in D$, so that $\xi \hat{\star} \eta=\xi \star \eta$.

3. Since

$$
\begin{aligned}
& \llbracket T \hat{\star} f, g \star \bar{h} \rrbracket=\left(\bar{T} \star_{1} h \mid f \star_{1} g\right)_{2}=\overline{\llbracket \bar{T} \star_{1} h, \bar{g} \star \bar{f} \rrbracket} \\
& =\overline{\llbracket \bar{T}, h \star(\bar{g} \star \bar{f}) \rrbracket}=\llbracket T,(f \star g) \star \bar{h} \rrbracket \\
& =\llbracket T, f \star(g \star \bar{h}) \rrbracket=\llbracket T \star_{1} f, g \star \bar{h} \rrbracket
\end{aligned}
$$

for all $g, h \in D$, the result follows.

4. This result follows from conjugating the preceding one.

5. Since

$$
\begin{aligned}
\llbracket S \hat{\star} T, g \star \bar{h} \rrbracket & =\frac{\left(\bar{S} \star_{1} h \mid T \star_{1} g\right)_{2}}{\llbracket \bar{\llbracket} \bar{S} \star_{1} h, \overline{T \star_{1} g} \rrbracket} \\
& =\overline{\llbracket \bar{S}, h \star \overline{\left(T \star_{1} g\right)} \rrbracket}=\llbracket S,\left(T \star_{1} g\right) \star \bar{h} \rrbracket \\
& =\llbracket S, T \star_{1}(g \star \bar{h}) \rrbracket
\end{aligned}
$$

for all $g, h \in D$, we see that $\llbracket S \hat{\star} T, f \rrbracket=\llbracket S, T \star_{1} f \rrbracket$ for all $f \in D$.

6. Now

$$
\begin{aligned}
\llbracket(S \hat{\star} T) \star_{1} f, g \rrbracket & =\llbracket S \hat{\star} T, f \star g \rrbracket=\llbracket S, T \star_{1}(f \star g) \rrbracket \\
& =\llbracket S,\left(T \star_{1} f\right) \star g \rrbracket=\llbracket S \star_{1}\left(T \star_{1} f\right), g \rrbracket
\end{aligned}
$$

for $f, g \in D$, so $(S \hat{\star} T) \star_{1} f=S \star_{1}\left(T \star_{1} f\right) \in D$ for all $f \in D$, which implies that $S \hat{\star} T \in N$. Moreover $\overline{S \hat{\star} T}=\bar{T} \hat{\star} \bar{S} \in N$, so $S \hat{\star} T \in \bar{N}$ as well.

The distribution $1 \in D^{\prime}$ will now be shown to be a left and right identity for $\hat{\star}$.

Lemma 5.3. The distribution $1=\overline{1}$ belongs to $D_{b}^{\prime} \cap N$, and $1 \hat{\star} T=T$, $S \hat{\star} 1=S$ for all $T \in Q, S \in \bar{Q}$. Moreover $\hat{\pi}(1)=\operatorname{id}_{H}, \pi_{0}(1)=\operatorname{id}_{H_{\Omega}}$, $\pi(1)=\operatorname{id}_{D}$. 
Proof. It is clear that $1 \star_{1} f=f \star_{2} 1=f$ for all $f \in D$, and hence $1=\overline{1}$ belongs to $N \cap \bar{N}$. If $T \in Q$, then

$$
\llbracket 1 \hat{\star} T, f \star \bar{g} \rrbracket=\left(g \mid T \star_{1} f\right)_{2}=\llbracket T \star_{1} f, \bar{g} \rrbracket=\llbracket T, f \star \bar{g} \rrbracket
$$

for all $f, g \in D$, and hence $1 \hat{\star} T=T$ for all $T \in Q$. That $S \hat{\star} 1=S$ for all $S \in \bar{Q}$ follows by conjugation. It is now immediate that $\pi(1)=\operatorname{id}_{D}$.

Since $|\llbracket 1, f \star \bar{g} \rrbracket|=\left|(g \mid f)_{2}\right| \leq\|f\|_{2} \cdot\|g\|_{2} \leq 1$ for all $f, g \in D$ with $\|f\|_{2},\|g\|_{2} \leq 1$, we deduce that $1 \in D_{b}^{\prime}$ with $\|1\| \leq 1$. Since $\pi(1) \leq \hat{\pi}(1)$, we deduce that $\hat{\pi}(1)=\operatorname{id}_{H}$ and $\pi_{0}(1)=\operatorname{id}_{H_{\Omega}}$.

It is crucial to remain aware that the $\hat{\star}$-product is not associative in general, but it is in certain limited cases.

Lemma 5.4. The following are true:

1. If $S \in \bar{N}, T \in N$ and $R \in Q \cap \bar{Q}$ then $S \hat{\star} R \in \bar{Q}, R \hat{\star} T \in Q$, and $(S \hat{\star} R) \hat{\star} T=S \hat{\star}(R \hat{\star} T)$.

2. If $S \in \bar{Q}, T \in Q$ and $R \in D_{b}^{\prime}$ then $S \star R \in \bar{Q}, R \hat{\star} T \in Q$ and $(S \hat{\star} R) \hat{\star} T=$ $S \hat{\star}(R \hat{\star} T)$.

Proof.

1. Since

$$
\begin{aligned}
\llbracket(R \hat{\star} T) \star_{1} f, g \rrbracket & =\llbracket R \hat{\star} T, f \star g \rrbracket=\left(\bar{R} \star_{1} \bar{g} \mid T \star_{1} f\right)_{2} \\
& =\overline{\llbracket \bar{R} \star_{1} \bar{g}, \overline{T \star_{1} f} \rrbracket}=\overline{\llbracket \bar{R}, \bar{g} \star\left(\overline{T \star_{1} f}\right) \rrbracket} \\
& =\llbracket R,\left(T \star_{1} f\right) \star g \rrbracket=\llbracket R \star_{1}\left(T \star_{1} f\right), g \rrbracket
\end{aligned}
$$

for $f, g \in D$, we see that $(R \hat{\star} T) \star_{1} f=R \star_{1}\left(T \star_{1} f\right) \in H$ for all $f \in D$, which implies that $R \star T \in Q$. By conjugation, we deduce that $S \hat{\star} R \in \bar{Q}$ as well. In addition

$$
\begin{aligned}
\llbracket S \hat{\star}(R \hat{\star} T), f \star \bar{g} \rrbracket & =\left(\bar{S} \star_{1} g \mid(R \hat{\star} T) \star_{1_{1}} f\right)_{2}=\left(\bar{S} \star_{1} g \mid R \star_{1}\left(T \star_{1} f\right)\right)_{2} \\
& =\llbracket R \star_{1}\left(T \star_{1} f\right), \overline{\bar{S} \star_{1} g} \rrbracket=\llbracket R,\left(T \star_{1} f\right) \star\left(\bar{S} \star_{1} g\right) \rrbracket \\
& =\overline{\llbracket \bar{R},\left(\bar{S} \star_{1} g\right) \star\left(\overline{T \star_{1} f}\right) \rrbracket}=\overline{\llbracket \bar{R} \star_{1}\left(\bar{S} \star_{1} g\right), \overline{T \star_{1} f} \rrbracket} \\
& =\llbracket(\bar{R} \hat{\star} \bar{S}) \star_{1} g, \overline{T \star_{1} f} \rrbracket=\left(\overline{(S \star R)} \star_{1} g \mid T \star_{1} f\right)_{2} \\
& =\llbracket(S \star R) \star T, f \star \bar{g} \rrbracket
\end{aligned}
$$

for any $f, g \in D$, which shows that $S \hat{\star}(R \hat{\star} T)=(S \hat{\star} R) \hat{\star} T$, as required. 
2. Since

$$
\begin{aligned}
\llbracket(R \hat{\star} T) \star_{1} f, g \rrbracket & =\llbracket R \hat{\star} T, f \star g \rrbracket=\left(\bar{R} \star_{1} \bar{g} \mid T \star_{1} f\right)_{2} \\
& =(\pi(\bar{R}) \bar{g} \mid \pi(T) f)_{2}=\left(\hat{\pi}(R)^{\star} \bar{g} \mid \pi(T) f\right)_{2} \\
& =(\bar{g} \mid \hat{\pi}(R) \pi(T) f)_{2}=\llbracket \hat{\pi}(R) \pi(T) f, g \rrbracket
\end{aligned}
$$

for any $f, g \in D$, we see that $(R \hat{\star} T) \star_{1} f=\hat{\pi}(R) \pi(T) f \in H$ for any $f \in D$, and hence that $R \hat{\star} T \in Q$. By conjugation, we deduce that $S \hat{\star} R \in \bar{Q}$ as well. Moreover

$$
\begin{aligned}
\llbracket S \hat{\star}(R \hat{\star} T), f \star \bar{g} \rrbracket & =\left(\bar{S} \star_{1} g \mid(R \hat{\star} T) \star_{1} f\right)_{2}=(\pi(\bar{S}) g \mid \hat{\pi}(R) \pi(T) f)_{2} \\
& =\left(\hat{\pi}(R)^{\star} \pi(\bar{S}) g \mid \pi(T) f\right)_{2}=(\hat{\pi}(\bar{R}) \pi(\bar{S}) g \mid \pi(T) f)_{2} \\
& =\left((\bar{R} \hat{\star} \bar{S}) \star_{1} g \mid T \star_{1} f\right)_{2}=\left((S \hat{\star} R) \star_{1} g \mid T \star_{1} f\right)_{2} \\
& =\llbracket(S \hat{\star} R) \hat{\star} T, f \star \bar{g} \rrbracket
\end{aligned}
$$

for $f, g \in D$, showing that $S \hat{\star}(R \hat{\star} T)=(S \hat{\star} R) \hat{\star} T$, as required.

Thus we know that, with respect to this product $\hat{\star}$, the space of bounded distributions $D_{b}^{\prime}$ is a unital associative *-algebra (indeed, it is a $C^{*}$-algebra). The results above show us that the same is true of the space $N \cap \bar{N}$. On the other hand, while the space $Q \cap \bar{Q}$ is *-invariant, and it is possible to define a bilinear map $\hat{\star}:(Q \cap \bar{Q}) \times(Q \cap \bar{Q}) \rightarrow D^{\prime}$, it is not true that the image of this map is a subspace of $Q \cap \bar{Q}$, nor is it the case that this product is associative, even when the product of three distributions can be calculated in both ways.

However, bearing these concerns about associativity in mind, we now have enough structure to study the arguments in [9] concerning the spectral properties of maps obtained using this product. We shall not need to restrict attention to the "easy" space $N \cap \bar{N}$ - our analysis works well in $Q \cap \bar{Q}$. Note that a distribution $T \in Q \cap \bar{Q}$ corresponds to an unbounded linear operator $\pi(T)$ on $H_{\Omega}$ with domain $D_{\Omega}$ which is adjointable and which possesses an adjoint whose domain contains $D_{\Omega}$. In other words, distributions in $Q \cap \bar{Q}$ correspond to unbounded linear operators belonging to the op-*-algebra $\mathcal{L}^{+}\left(D_{\Omega}, H_{\Omega}\right),[4,22]$.

\section{$\S 6$. Distribution-Valued Spectral Theory}

In [9], attempts are made to define resolvent distributions, and hence derive a spectral calculus, for "real" distributions $T=\bar{T} \in N \cap \bar{N}$. However, these calculations seem to be based on some mistaken assumptions concerning 
the associativity of the product $\hat{\star}$. In the following section we shall clarify the situation. Our approach will be the same as that in [9], so that a general "real" distribution will be approximated by a sequence of bounded "real" distributions.

For the remainder of the paper we shall reserve the symbol $T$ for an arbitrarily chosen, but then fixed "real" element of $Q \cap \bar{Q}$. It is clear from previous analysis that $\pi(T)$ is a symmetric element of $\mathcal{L}^{+}\left(D_{\Omega}, H_{\Omega}\right)$. We shall also reserve the symbol $y$ for a freely chosen nonzero real number. For any self-adjoint operator, iy belongs to its resolvent set.

We begin by defining a sequence of distributions that are central to the construction of sequences converging to candidate resolvents for $T$ acting as an operator.

Lemma 6.1. For any $k \geq 0$, define

$$
P_{k}=\sum_{n=0}^{k} a_{n n} .
$$

Then $P_{k}$ has the following properties.

1. $P_{k}=\overline{P_{k}}=P_{k} \star P_{k}$ is an element of $D$;

2. for any $\xi=\sum_{m, n \geq 0} \xi_{m n} a_{m n} \in H$,

$$
\begin{aligned}
P_{k} \star \xi & =\sum_{m=0}^{k} \sum_{n \geq 0} \xi_{m n} a_{m n} \\
\xi \star P_{k} & =\sum_{m \geq 0} \sum_{n=0}^{k} \xi_{m n} a_{m n} \\
P_{k} \star \xi \star P_{k} & =\sum_{m, n=0}^{k} \xi_{m n} a_{m n},
\end{aligned}
$$

with $P_{k} \star \xi \star P_{k} \in D$.

3. Each sequence $\left(P_{k} \star \xi\right)_{k},\left(\xi \star P_{k}\right)_{k},\left(P_{k} \star \xi \star P_{k}\right)_{k}$ converges in $H$, as $k \rightarrow \infty$, to $\xi$ for any $\xi \in H$,

4. Each sequence $\left(P_{k} \star f\right)_{k},\left(f \star P_{k}\right)_{k},\left(P_{k} \star f \star P_{k}\right)_{k}$ converges in $D$, as $k \rightarrow \infty$, to $f$ for any $f \in D$. 
5. The vectors

$$
\begin{aligned}
& P_{k} \hat{\star} T=P_{k} \star_{2} T=\sum_{n=0}^{k} a_{n} \star \overline{\left(T \star_{1} a_{n}\right)}=\sum_{n=0}^{k} a_{n} \star \overline{\pi(T) a_{n}} \\
& T \hat{\star} P_{k}=T \star_{1} P_{k}=\sum_{n=0}^{k}\left(T \star_{1} a_{n}\right) \star \overline{a_{n}}=\sum_{n=0}^{k} \pi(T) a_{n} \star \overline{a_{n}}
\end{aligned}
$$

belong to $H$ for any $k \geq 0$.

Due to the above results, particularly the last item, we can act on $P_{k}$ and $P_{k} \hat{\star} T$ with $\hat{\pi}$ to obtain bounded operators on $H$.

Lemma 6.2. The operators

$$
\begin{aligned}
& A_{k}=\hat{\pi}\left(P_{k} \hat{\star} T\right), \\
& \Pi_{k}=\hat{\pi}\left(P_{k}\right)
\end{aligned}
$$

belong to $\mathcal{B}_{*}(H)$ for any $k \geq 0$.

The following properties result from these definitions. For any $k \geq 0$,

$$
\begin{aligned}
\Pi_{k} & =\Pi_{k}^{\star}=\Pi_{k}^{2} \\
A_{k}^{\star} & =\hat{\pi}\left(T \hat{\star} P_{k}\right) \\
\Pi_{k} A_{k}^{\star} & =A_{k} \Pi_{k} \\
\Pi_{k} A_{k} & =A_{k} \\
A_{k}^{\star} \Pi_{k} & =A_{k}^{\star} \\
\Pi_{k} A_{k} A_{k}^{\star} & =A_{k} A_{k}^{\star} \Pi_{k}=A_{k} A_{k}^{\star} \\
A_{k}^{\star} A_{k} & =\sum_{n=0}^{k} \hat{\pi}\left(\pi(T) a_{n}\right) \hat{\pi}\left(\pi(T) a_{n}\right)^{\star}
\end{aligned}
$$

Proposition 6.1. (a) There exists a "real" element $\mathcal{R}_{T, y}=\overline{\mathcal{R}_{T, y}}$ in $D_{b}^{\prime}$ such that the limit

$$
\left(y^{2} I+A_{k}^{\star} A_{k}\right)^{-1} \underset{k \rightarrow \infty}{\longrightarrow} \hat{\pi}\left(\mathcal{R}_{T, y}\right)
$$

holds in the strong operator topology.

(b) The positivity property

$$
0 \leq \hat{\pi}\left(\mathcal{R}_{T, y}\right) \leq y^{-2} I
$$

holds, ensuring that

$$
\left\|\mathcal{R}_{T, y}\right\| \leq y^{-2}
$$


(c) Both $\mathcal{R}_{T, y} \hat{\star} T$ and $T \hat{\star} \mathcal{R}_{T, y}$ belong to $D_{b}^{\prime}$, with

$$
\left\|\mathcal{R}_{T, y} \hat{\star} T\right\|,\left\|T \hat{\star} \mathcal{R}_{T, y}\right\| \leq|y|^{-1} .
$$

(d) The limit

$$
\left(y^{2} I+A_{k}^{\star} A_{k}\right)^{-1} A_{k}^{\star} \underset{k \rightarrow \infty}{\rightarrow} \hat{\pi}\left(\mathcal{R}_{T, y} \hat{\star} T\right)
$$

holds in the strong operator topology, while the limit

$$
A_{k}\left(y^{2} I+A_{k}^{\star} A_{k}\right)^{-1} \underset{k \rightarrow \infty}{\rightarrow} \hat{\pi}\left(T \hat{\star} \mathcal{R}_{T, y}\right)
$$

holds in the weak operator topology.

Proof. (a) Now $\left(\left(y^{2} I+A_{k}^{\star} A_{k}\right)\right)_{k \geq 0}$ is an increasing sequence in $\mathcal{B}_{\star}(H)_{+}$, so that $\left(\left(y^{2} I+A_{k}^{\star} A_{k}\right)^{-1}\right)_{k>0}$ is a decreasing sequence in $\mathcal{B}_{*}(H)_{+}$, with $0 \leq$ $\left(y^{2} I+A_{k}^{\star} A_{k}\right)^{-1} \leq y^{-2} I$ for all $k \geq 0$. This proves the existence of $\mathcal{R}_{T, y}$, and (b) is immediate.

(c) Since

$$
\begin{aligned}
\llbracket \mathcal{R}_{T, y} \hat{\star} T, f \star \bar{g} \rrbracket & =\left(\hat{\pi}\left(\mathcal{R}_{T, y}\right) g \mid T \star_{1} f\right)_{2}=\lim _{k \rightarrow \infty}\left(\left(y^{2} I+A_{k}^{\star} A_{k}\right)^{-1} g \mid T \star_{1} f\right)_{2} \\
& =\lim _{k \rightarrow \infty}\left(\left(y^{2} I+A_{k}^{\star} A_{k}\right)^{-1} g \mid T \star_{1}\left(P_{k} \star f\right)\right)_{2} \\
& =\lim _{k \rightarrow \infty}\left(\left(y^{2} I+A_{k}^{\star} A_{k}\right)^{-1} g \mid\left(T \star_{1} P_{k}\right) \star_{1} f\right)_{2} \\
& =\lim _{k \rightarrow \infty}\left(g \mid\left(y^{2} I+A_{k}^{\star} A_{k}\right)^{-1} A_{k}^{\star} f\right)_{2}
\end{aligned}
$$

for any $f, g \in D$, and $\left\|\left(y^{2} I+A_{k}^{\star} A_{k}\right)^{-1} A_{k}^{\star}\right\|_{2} \leq|y|^{-1}$ for all $k \geq 0$, it follows that

$$
\left|\llbracket \mathcal{R}_{T, y} \hat{\star} T, f \star \bar{g} \rrbracket\right| \leq|y|^{-1}\|f\|_{2} \cdot\|g\|_{2}
$$

for all $f, g \in D$. Thus $\mathcal{R}_{T, y} \hat{\star} T \in D_{b}^{\prime}$, with $\left\|\mathcal{R}_{T, y} \hat{\star} T\right\| \leq|y|^{-1}$. In addition $T \hat{\star} \mathcal{R}_{T, y}=\overline{\mathcal{R}_{T, y} \hat{\star} T}$, so $T \hat{\star} \mathcal{R}_{T, y} \in D_{b}^{\prime}$, with $\left\|T \hat{\star} \mathcal{R}_{T, y}\right\| \leq|y|^{-1}$.

(d) Since we have just shown that

$$
\left(g \mid \hat{\pi}\left(\mathcal{R}_{T, y} \hat{\star} T\right) f\right)_{2}=\lim _{k \rightarrow \infty}\left(g \mid\left(y^{2} I+A_{k}^{\star} A_{k}\right)^{-1} A_{k}^{\star} f\right)_{2}
$$

for any $f, g \in D$, a density argument shows that $\left(y^{2} I+A_{k}^{\star} A_{k}\right)^{-1} A_{k}^{\star} \underset{k \rightarrow \infty}{\longrightarrow}$ $\hat{\pi}\left(\mathcal{R}_{T, y} \hat{\star} T\right)$ in the weak operator topology. Since

$$
\begin{aligned}
& \left\|\left(y^{2} I+A_{k}^{\star} A_{k}\right)^{-1} A_{k}^{\star} f-\hat{\pi}\left(\mathcal{R}_{T, y} \hat{\star} T\right) f\right\|_{2} \\
& \leq\left\|\left(y^{2} I+A_{k}^{\star} A_{k}\right)^{-1}\left(A_{k}^{\star} f-T \star_{1} f\right)\right\|_{2}+\left\|\left[\left(y^{2} I+A_{k}^{\star} A_{k}\right)^{-1}-\hat{\pi}\left(\mathcal{R}_{T, y}\right)\right]\left(T \star_{1} f\right)\right\|_{2} \\
& \leq|y|^{-2}\left\|\pi(T)\left[P_{k} \star f-f\right]\right\|_{2}+\left\|\left[\left(y^{2} I+A_{k}^{\star} A_{k}\right)^{-1}-\hat{\pi}\left(\mathcal{R}_{T, y}\right)\right]\left(T \star_{1} f\right)\right\|_{2}
\end{aligned}
$$


for any $k \geq 0$ and $f \in D$. This implies that $\left(y^{2} I+A_{k}^{\star} A_{k}\right)^{-1} A_{k}^{\star} f \underset{k \rightarrow \infty}{\rightarrow}$ $\hat{\pi}\left(\mathcal{R}_{T, y} \hat{\star} T\right) f$ in $H$ for any $f \in D$. By another density argument, $\left(y^{2} I+\right.$ $\left.A_{k}^{\star} A_{k}\right)^{-1} A_{k}^{\star} \underset{k \rightarrow \infty}{\rightarrow} \hat{\pi}\left(\mathcal{R}_{T, y} \hat{\star} T\right)$ in the strong operator topology.

Given the above strong convergence result, it is immediate that

$$
A_{k}\left(y^{2} I+A_{k}^{\star} A_{k}\right)^{-1} \underset{k \rightarrow \infty}{\rightarrow} \hat{\pi}\left(\mathcal{R}_{T, y} \hat{\star} T\right)^{\star}=\hat{\pi}\left(T \hat{\star} \mathcal{R}_{T, y}\right)
$$

in the weak operator topology.

The observation that one of these sequences converges in the strong operator topology, while the second appears only to converge in the weak operator topology, (while being consistent with the known fact that adjunction is not strong operator continuous) begins to show the difficulties that will follow. That there is a difference between these two sequences is, in part, a consequence of the lack of symmetry in the manner of defining $A_{k}$ from $T$. This sort of asymmetry will have major implications. Some sequences will converge in certain topologies, while other similar sequences will not. Some operators will commute with the projection operators $\Pi_{k}$, while other similar operators will not. The difficulties that these differences cause can be associated with the lack of associativity of the Moyal product. In order to attempt to create a spectral theory for unbounded symmetric distributions, we need to push the domain of the Moyal product to its limit, perhaps beyond in some sense, and hence difficulties of this type are to be expected.

On the basis of our procedures earlier, we expect that in order to proceed we will have to define a second resolvent candidate to associate with left $\hat{\star}$ multiplication by $T$. But note carefully, the defining sequence for it converges in the weak operator topology only: we cannot improve this to strong operator convergence with the second resolvent candidate.

Proposition 6.2. There exists a "real" distribution $\hat{\mathcal{R}}_{T, y}=\overline{\hat{\mathcal{R}}_{T, y}}$ in $D_{b}^{\prime}$ such that

$$
\left(y^{2} I+A_{k} A_{k}^{\star}\right)^{-1} \underset{k \rightarrow \infty}{\rightarrow} \hat{\pi}\left(\hat{\mathcal{R}}_{T, y}\right)
$$

in the weak operator topology. Moreover

$$
\begin{aligned}
\left\|T \hat{\star}\left(\mathcal{R}_{T, y} \hat{\star} T\right)\right\| & \leq 1, \\
\left\|\hat{\mathcal{R}}_{T, y}\right\| & \leq|y|^{-2}, \\
y^{2} \hat{\mathcal{R}}_{T, y}+T \hat{\star}\left(\mathcal{R}_{T, y} \hat{\star} T\right) & =1 .
\end{aligned}
$$


Proof. Since $A_{k}\left(y^{2} I+A_{k}^{\star} A_{k}\right)^{-1}=\left(y^{2} I+A_{k} A_{k}^{\star}\right)^{-1} A_{k}$ for any $k \geq 0$, we see that

$$
y^{2}\left(y^{2} I+A_{k} A_{k}^{\star}\right)^{-1}+A_{k}\left(y^{2} I+A_{k}^{\star} A_{k}\right)^{-1} A_{k}^{\star}=I
$$

for $k \geq 0$, yielding positive bounded maps $\left(y^{2} I+A_{k} A_{k}^{\star}\right)^{-1} \leq y^{-2} I$ and $A_{k}\left(y^{2} I+A_{k}^{\star} A_{k}\right)^{-1} A_{k}^{\star} \leq I$ for all $k \geq 0$. Then

$$
\begin{aligned}
& \left(g \mid A_{k}\left(y^{2} I+A_{k}^{\star} A_{k}\right)^{-1} A_{k}^{\star} f\right)_{2} \\
& \quad=\left(A_{k}^{\star} g \mid\left(y^{2} I+A_{k}^{\star} A_{k}\right)^{-1} A_{k}^{\star} f\right)_{2}=\left(T \star_{1}\left(P_{k} \star g\right) \mid\left(y^{2} I+A_{k}^{\star} A_{k}\right)^{-1} A_{k}^{\star} f\right)_{2} \\
& \underset{k \rightarrow \infty}{\rightarrow}\left(T \star_{1} g \mid \hat{\pi}\left(\mathcal{R}_{T, y} \hat{\star} T\right) f\right)_{2}=\left(T \star_{1} g \mid\left(\mathcal{R}_{T, y} \hat{\star} T\right) \star_{1} f\right)_{2} \\
& =\llbracket T \hat{\star}\left(\mathcal{R}_{T, y} \hat{\star} T\right), f \star \bar{g} \rrbracket
\end{aligned}
$$

for all $f, g \in D$. This implies that $T \hat{\star}\left(\mathcal{R}_{T, y} \hat{\star} T\right)=\left(T \hat{\star} \mathcal{R}_{T, y}\right) \hat{\star} T$ belongs to $D_{b}^{\prime}$, and that

$$
A_{k}\left(y^{2} I+A_{k}^{\star} A_{k}\right)^{-1} A_{k}^{\star} \underset{k \rightarrow \infty}{\rightarrow} \hat{\pi}\left(T \hat{\star}\left(\mathcal{R}_{T, y} \hat{\star} T\right)\right)
$$

in the weak operator topology. Thus we deduce that $\left(y^{2} I+A_{k} A_{k}^{*}\right)^{-1}$ converges, as $k \rightarrow \infty$, in the weak operator topology to $\hat{\pi}\left(\hat{\mathcal{R}}_{T, y}\right)$ for some well-defined bounded distribution $\hat{\mathcal{R}}_{T, y}$. The rest of this corollary is now immediate.

When proving the existence of $\mathcal{R}_{T, y}$, it was possible to appeal to the increasing nature of the sequence $\left(A_{k}^{\star} A_{k}\right)_{k}$ to derive the strong operator convergence of $\left(\left(y^{2} I+A_{k}^{\star} A_{k}\right)^{-1}\right)_{k}$ to $\hat{\pi}\left(\mathcal{R}_{T, y}\right)$. Since, on the other hand, the sequence $\left(A_{k} A_{k}^{\star}\right)_{k}$ is not increasing, no such argument is possible, and hence the argument establishing the existence of $\hat{\mathcal{R}}_{T, y}$ is more indirect, and yields weak operator convergence only. This is another (and key) example of the way in which small differences in the formalism will have a substantial impact on the analysis. The sequence $\left(\left(y^{2} I+A_{k}^{\star} A_{k}\right)^{-1}\right)_{k}$ converges in the strong operator topology, but its elements do not commute with $\Pi_{k}$. On the other hand, the sequence $\left(\left(y^{2} I+A_{k} A_{k}^{\star}\right)^{-1}\right)_{k}$ only seems to converge in the weak operator topology, but its elements commute with $\Pi_{k}$. Life would be much simpler if both properties were satisfied by both sequences! It is worth noting that some of the problems that arise in [9] are due to the fact that the distributions $\mathcal{R}_{T, y}$ and $\hat{\mathcal{R}}_{T, y}$ have not there been recognized as being different.

Proposition 6.3. $\quad$ The following properties of $\mathcal{R}_{T, y}$ and $\hat{\mathcal{R}}_{T, y}$ can be established:

1. $T \hat{\star} \hat{\mathcal{R}}_{T, y}=\mathcal{R}_{T, y} \hat{\star} T$ and $T \hat{\star} \mathcal{R}_{T, y}=\hat{\mathcal{R}}_{T, y} \hat{\star} T$, 
2. $\left(\mathcal{R}_{T, y} \hat{\star} T\right) \hat{\star} T=1-y^{2} \mathcal{R}_{T, y}$,

3. $\left.\left(\hat{\mathcal{R}}_{T, y} \hat{\star} T\right)\right) \hat{\star} T=1-y^{2} \hat{\mathcal{R}}_{T, y}$,

4. $y^{2} \mathcal{R}_{T, y}+T \hat{\star}\left(\hat{\mathcal{R}}_{T, y} \hat{\star} T\right)=1$,

5. $\left\|T \hat{\star} \hat{\mathcal{R}}_{T, y}\right\|,\left\|\hat{\mathcal{R}}_{T, y} \hat{\star} T\right\| \leq|y|^{-1}$,

6. The distribution $T \hat{\star}\left(\hat{\mathcal{R}}_{T, y} \hat{\star} T\right)=\left(T \hat{\star} \hat{\mathcal{R}}_{T, y}\right) \hat{\star} T$ belongs to $D_{b}^{\prime}$, with $\left\|T \hat{\star}\left(\hat{\mathcal{R}}_{T, y} \hat{\star} T\right)\right\| \leq 1$.

Proof.

1. Since

$$
\begin{aligned}
& \llbracket T \hat{\star} \hat{\mathcal{R}}_{T, y}, f \star \bar{g} \rrbracket \\
& =\left(T \star_{1} g \mid \hat{\mathcal{R}}_{T, y} \star_{1} f\right)_{2}=\lim _{k \rightarrow \infty}\left(T \star_{1} g \mid\left(y^{2} I+A_{k} A_{k}^{\star}\right)^{-1} f\right)_{2} \\
& =\lim _{k \rightarrow \infty}\left(P_{k} \star\left(T \star_{1} g\right) \mid\left(y^{2} I+A_{k} A_{k}^{\star}\right)^{-1} f\right)_{2} \\
& =\lim _{k \rightarrow \infty}\left(A_{k} g \mid\left(y^{2} I+A_{k} A_{k}^{\star}\right)^{-1} f\right)_{2} \\
& =\lim _{k \rightarrow \infty}\left(g \mid A_{k}^{\star}\left(y^{2} I+A_{k} A_{k}^{\star}\right)^{-1} f\right)_{2}=\lim _{k \rightarrow \infty}\left(g \mid\left(y^{2} I+A_{k}^{\star} A_{k}\right)^{-1} A_{k}^{\star} f\right)_{2} \\
& =\left(g \mid \hat{\pi}\left(\mathcal{R}_{T, y} \hat{\star} T\right) f\right)_{2}=\llbracket \mathcal{R}_{T, y} \hat{\star} T, f \star \bar{g} \rrbracket
\end{aligned}
$$

for all $f, g \in D$, we see that $T \hat{\star} \hat{\mathcal{R}}_{T, y}=\mathcal{R}_{T, y} \hat{\star} T$. Conjugation then gives us that $T \hat{\star} \mathcal{R}_{T, y}=\hat{\mathcal{R}}_{T, y} \hat{\star} T$.

2. Since

$$
\begin{aligned}
& \llbracket\left(\mathcal{R}_{T, y} \hat{\star} T\right) \hat{\star} T, f \star \bar{g} \rrbracket \\
& =\left(\left(\left(\mathcal{R}_{T, y} \hat{\star} T\right) \star_{1} g \mid T \star_{1} f\right)_{2}=\left(\hat{\pi}\left(\mathcal{R}_{T, y} \hat{\star} T\right)^{\star} g \mid T \star_{1} f\right)_{2}\right. \\
& =\left(g \mid \hat{\pi}\left(\mathcal{R}_{T, y} \hat{\star} T\right)\left(T \star_{1} f\right)\right)_{2}=\lim _{k \rightarrow \infty}\left(g \mid\left(y^{2} I+A_{k}^{\star} A_{k}\right)^{-1} A_{k}^{\star}\left(T \star_{1} f\right)\right)_{2} \\
& =\lim _{k \rightarrow \infty}\left(g \mid\left(y^{2} I+A_{k}^{\star} A_{k}\right)^{-1} A_{k}^{\star} A_{k} f\right)_{2} \\
& =\lim _{k \rightarrow \infty}\left(g \mid\left(I-y^{2}\left(y^{2} I+A_{k}^{\star} A_{k}\right)^{-1}\right) f\right)_{2} \\
& =\left(g \mid f-y^{2} \hat{\pi}\left(\mathcal{R}_{T, y}\right) f\right)_{2}=\llbracket 1-y^{2} \mathcal{R}_{T, y}, f \star \bar{g} \rrbracket
\end{aligned}
$$

for all $f, g \in D$, it follows that $\left(\mathcal{R}_{T, y} \hat{\star} T\right) \hat{\star} T=1-y^{2} \mathcal{R}_{T, y}$.

3. $\left(\hat{\mathcal{R}}_{T, y} \hat{\star} T\right) \hat{\star} T=\left(T \hat{\star} \mathcal{R}_{T, y}\right) \hat{\star} T=T \hat{\star}\left(\mathcal{R}_{T, y} \hat{\star} T\right)=1-y^{2} \hat{\mathcal{R}}_{T, y}$. 
4. This follows since

$$
\begin{aligned}
y^{2} \mathcal{R}_{T, y}+T \hat{\star}\left(\hat{\mathcal{R}}_{T, y} \hat{\star} T\right) & =y^{2} \mathcal{R}_{T, y}+\left(T \hat{\star} \hat{\mathcal{R}}_{T, y}\right) \hat{\star} T \\
& =y^{2} \mathcal{R}_{T, y}+\left(\mathcal{R}_{T, y} \hat{\star} T\right) \hat{\star} T=1 .
\end{aligned}
$$

5. This is immediate from the first part, and the known properties of the distribution $\mathcal{R}_{T, y}$.

6. Since

$$
T \hat{\star}\left(\hat{\mathcal{R}}_{T, y} \hat{\star} T\right)=\left(T \hat{\star} \hat{\mathcal{R}}_{T, y}\right) \hat{\star} T=\left(\mathcal{R}_{T, y} \hat{\star} T\right) \hat{\star} T=1-y^{2} \mathcal{R}_{T, y},
$$

we see that the distribution $X=T \hat{\star}\left(\hat{\mathcal{R}}_{T, y} \hat{\star} T\right)$ belongs to $D_{b}^{\prime}$, and that $0 \leq \hat{\pi}(X) \leq I$, which certainly implies that $\|X\| \leq 1$.

In summary, then, we have found two similar but (as we shall see) distinct distributions. Note that the particular distributions $\mathcal{R}_{T, 1}$ and $\hat{\mathcal{R}}_{T, 1}$ can be seen as corresponding to what is written as $\left(1+T^{2}\right)^{-1}$ in [9].

Each of these two distributions can be used to define candidate resolvent operators, and so we define the tempered distribution-valued functions $\mathcal{S}$ and $\hat{\mathcal{S}}$ as follows:

$$
\begin{aligned}
& \mathcal{S}_{T, y}=\mathcal{R}_{T, y} \hat{\star}(T+i y 1), \\
& \hat{\mathcal{S}}_{T, y}=\hat{\mathcal{R}}_{T, y} \hat{\star}(T+i y 1) .
\end{aligned}
$$

Proposition 6.4. Both $\mathcal{S}_{T, y}$ and $\hat{\mathcal{S}}_{T, y}$ are bounded one-sided inverses for $T-i y 1$, in that $\left\|\mathcal{S}_{T, y}\right\|,\left\|\hat{\mathcal{S}}_{T, y}\right\| \leq 2|y|^{-1}$, and $\mathcal{S}_{T, y} \hat{\star}(T-i y 1)=$ $\hat{\mathcal{S}}_{T, y} \hat{\star}(T-i y 1)=1$.

Proof. Since $\mathcal{S}_{T, y}=\mathcal{R}_{T, y} \hat{\star} T+i y \mathcal{R}_{T, y}$, it is clear that $\mathcal{S}_{T, y} \in D_{b}^{\prime}$ with norm bounded by $2|y|^{-1}$. We observe that

$$
\begin{aligned}
\mathcal{S}_{T, y} \hat{\star}(T-i y 1) & =\mathcal{S}_{T, y} \hat{\star} T-i y \mathcal{S}_{T, y} \\
& =\left[\mathcal{R}_{T, y} \hat{\star}(T+i y 1)\right] \hat{\star} T-i y \mathcal{R}_{T, y} \hat{\star}(T+i y 1) \\
& =\left(\mathcal{R}_{T, y} \hat{\star} T\right) \hat{\star} T+i y \mathcal{R}_{T, y} \hat{\star} T-i y \mathcal{R}_{T, y} \hat{\star} T+y^{2} \mathcal{R}_{T, y} \\
& =1 .
\end{aligned}
$$

The results for $\hat{\mathcal{S}}_{T, y}$ are proved similarly. 
In general, neither of these distributions is a two-sided inverse for $T-i y 1$, which complicates matters. However, we can combine them to find a two-sided inverse. Define

$$
z_{T, y}=\frac{1}{2}\left[\mathcal{S}_{T, y}+\hat{\mathcal{S}}_{T, y}\right]
$$

Proposition 6.5. The distribution $z_{T, y}$ is bounded, with $\left\|z_{T, y}\right\| \leq$ $2|y|^{-1}$. Moreover $z_{T, y}$ is a two-sided inverse for $T-i y 1$. Finally $\frac{z_{T, y}}{z}=$ $z_{T,-y}$

Proof. It is clear that $z_{T, y} \in D_{b}^{\prime}$ with the given norm bound, and it is also immediate that

$$
z_{T, y} \hat{\star}(T-i y 1)=1
$$

Now

$$
z_{T, y}=\frac{1}{2}\left[\mathcal{R}_{T, y} \hat{\star} T+\hat{\mathcal{R}}_{T, y} \hat{\star} T+i y \mathcal{R}_{T, y}+i y \hat{\mathcal{R}}_{T, y}\right],
$$

which implies that

$$
\begin{aligned}
\overline{Z_{T, y}} & =\frac{1}{2}\left[T \hat{\star} \mathcal{R}_{T, y}+T \hat{\star} \hat{\mathcal{R}}_{T, y}-i y \mathcal{R}_{T, y}-i y \hat{\mathcal{R}}_{T, y}\right] \\
& =\frac{1}{2}\left[\hat{\mathcal{R}}_{T, y} \hat{\star} T+\mathcal{R}_{T, y} \hat{\star} T-i y \mathcal{R}_{T, y}-i y \hat{\mathcal{R}}_{T, y}\right] \\
& =z_{T,-y},
\end{aligned}
$$

since $\mathcal{R}_{T,-y}=\mathcal{R}_{T, y}$ and $\hat{\mathcal{R}}_{T,-y}=\hat{\mathcal{R}}_{T, y}$. Then we deduce that $\overline{z_{T, y}} \hat{\star}(T+i y 1)=$ 1 and hence, taking conjugates, that $(T-i y 1) \hat{\star} z_{T, y}=1$.

We now have two distributions which are something like $\left(y^{2} 1+T^{2}\right)^{-1}$, and three further distributions which seem to behave like distributional resolvents. It is important to understand the relationship between these distributions and the spectral properties of the unbounded symmetric operator $\pi(T)$. We start this process with the following technical result. It should be noted that this result is another special case where the product $\hat{\star}$ is associative, but it is the only result we have where a distribution as general as $T$ is the middle term.

Lemma 6.3. If $S \in D_{b}^{\prime}$ is such that $S \hat{\star} T \in D_{b}^{\prime}$ then $(S \hat{\star} T) \hat{\star} \hat{\mathcal{R}}_{T, y}=$ $S \hat{\star}\left(T \hat{\star} \hat{\mathcal{R}}_{T, y}\right)$.

Proof. Suppose $f \in D$ is such that $f=P_{\ell} \star f=f \star P_{\ell}$ for some $\ell \geq 0$. Such elements are dense in $D$. These elements have been used in Gayral et al, [7]. For any $k \geq \ell$, define

$$
f_{k}=\left(y^{2} I+A_{k} A_{k}^{*}\right)^{-1} f
$$


Since $\left[\Pi_{k}, A_{k} A_{k}^{*}\right]=0$ and $\Pi_{k} f=f$ for all $k \geq \ell$, we see that $\Pi_{k} f_{k}=f_{k}$ for all $k \geq \ell$. Also $f_{k} \star P_{\ell}=f_{k}$ for all $k \geq \ell$, and hence $f_{k} \in D$ for all $k \geq \ell$, and

$$
f_{k} \underset{k \rightarrow \infty}{\rightarrow} \hat{\pi}\left(\hat{\mathcal{R}}_{T, y}\right) f
$$

weakly, while

$$
\begin{aligned}
& T \star_{1} f_{k}=T \star_{1}\left(\Pi_{k} f_{k}\right)=A_{k}^{*} f_{k}=\left(y^{2} I+A_{k}^{*} A_{k}\right)^{-1} A_{k}^{*} f \\
& \underset{k \rightarrow \infty}{\longrightarrow} \hat{\pi}\left(\mathcal{R}_{T, y} \hat{\star} T\right) f=\hat{\pi}\left(T \hat{\star} \hat{\mathcal{R}}_{T, y}\right) f
\end{aligned}
$$

in the norm of $H$. Thus we deduce that

$$
\begin{aligned}
& \llbracket S \hat{\star}\left(T \hat{\star} \hat{\mathcal{R}}_{T, y}\right), f \star \bar{g} \rrbracket \\
& =\left(\bar{S} \star_{1} g \mid\left(T \hat{\star} \hat{\mathcal{R}}_{T, y}\right) \star_{1} f\right)_{2}=\left(\hat{\pi}(S)^{*} g \mid \hat{\pi}\left(T \hat{\star} \hat{\mathcal{R}}_{T, y}\right) f\right)_{2} \\
& =\lim _{k \rightarrow \infty}\left(\hat{\pi}(S)^{*} g \mid T \star_{1} f_{k}\right)_{2}=\lim _{k \rightarrow \infty}\left(g \mid \hat{\pi}(S)\left(T \star_{1} f_{k}\right)\right)_{2} \\
& =\lim _{k \rightarrow \infty}\left(g \mid \hat{\pi}(S \hat{\star} T) f_{k}\right)_{2}=\lim _{k \rightarrow \infty}\left(\hat{\pi}(S \hat{\star} T)^{*} g \mid f_{k}\right)_{2} \\
& =\left(\overline{(S \hat{\star} T)} \star_{1} g \mid \hat{\pi}\left(\hat{\mathcal{R}}_{T, y}\right) f\right)_{2}=\left(\overline{(S \hat{\star} T)} \star_{1} g \mid \hat{\mathcal{R}}_{T, y} \star_{1} f\right)_{2} \\
& =\llbracket(S \hat{\star} T) \hat{\star} \hat{\mathcal{R}}_{T, y}, f \star \bar{g} \rrbracket
\end{aligned}
$$

and the result now follows from standard density arguments.

We now obtain our first result connecting the distributions we have been studying and the associated spectral theory.

Lemma 6.4. The domain of the unbounded linear operator $\pi(T)^{*}$ is equal to

$$
\mathcal{D}\left(\pi(T)^{*}\right)=\{\phi \in H \mid T \hat{\star} \phi \in H\}
$$

with $\pi(T)^{*} \phi=T \hat{\star} \phi$ for all $\phi \in \mathcal{D}\left(\pi(T)^{*}\right)$. Moreover, the map $\pi(T)^{*}-i y I$ : $\mathcal{D}\left(\pi(T)^{*}\right) \rightarrow H$ is surjective.

Proof. If $\phi \in H$ and $f, g \in D$ then

$$
\begin{aligned}
(\pi(T)(f \star \bar{g}) \mid \phi)_{2} & =\left(T \star_{1}(f \star \bar{g}) \mid \phi\right)_{2}=\left(\left(T \star_{1} f\right) \star \bar{g} \mid \phi\right)_{2}=\left(R_{\bar{g}}\left(T \star_{1} f\right) \mid \phi\right)_{2} \\
& =\left(T \star_{1} f \mid R_{g} \phi\right)_{2}=\left(T \star_{1} f \mid \phi \star g\right)_{2}=\llbracket T \star \star \phi, g \star \bar{f} \rrbracket
\end{aligned}
$$

so that

$$
(\pi(T) f \mid \phi)_{2}=\llbracket T \hat{\star} \phi, \bar{f} \rrbracket \quad f \in D, \phi \in H
$$

Thus, if $\phi \in \mathcal{D}\left(\pi(T)^{*}\right)$, then $\left(f \mid \hat{\pi}(T)^{*} \phi\right)_{2}=\llbracket T \hat{\star} \phi, \bar{f} \rrbracket$ for all $f \in D$, so that $T \hat{\star} \phi=\hat{\pi}(T)^{*} \phi \in H$. Conversely, if $T \hat{\star} \phi \in H$ then $(\pi(T) f \mid \phi)_{2}=$ 
$(f \mid T \hat{\star} \phi)_{2}$ for all $f \in D$, so that $\phi$ does belong to $\mathcal{D}\left(\pi(T)^{*}\right)$, with $\hat{\pi}(T)^{*} \phi=$ $T \hat{\star} \phi$.

Now, if $\xi \in H$, then

$$
\begin{aligned}
\left(\pi(T) f \mid \hat{\pi}\left(\mathcal{Z}_{T, y}\right) \xi\right)_{2} & =\left(\hat{\pi}\left(\overline{Z_{T, y}}\right)\left(T \star_{1} f\right) \mid \xi\right)_{2}=\left(\hat{\pi}\left(z_{T,-y}\right)\left(T \star_{1} f\right) \mid \xi\right)_{2} \\
& =\left(\hat{\pi}\left(z_{T,-y} \hat{\star} T\right) f \mid \xi\right)_{2}=\left(\hat{\pi}\left(1-i y z_{T,-y}\right) f \mid \xi\right)_{2} \\
& =(f \mid \xi)_{2}+i y\left(f \mid \hat{\pi}\left(z_{T, y}\right) \xi\right)_{2}
\end{aligned}
$$

for all $f \in D$, so that $\hat{\pi}\left(z_{T, y}\right) \xi \in \mathcal{D}\left(\pi(T)^{*}\right)$ with

$$
\hat{\pi}(T)^{*}\left(\hat{\pi}\left(z_{T, y}\right) \xi\right)=\xi+i y \hat{\pi}\left(z_{T, y}\right) \xi
$$

which says that $\xi=\left[\pi(T)^{*}-i y I\right]\left(\hat{\pi}\left(\mathcal{Z}_{T, y}\right) \xi\right)$ belongs to the range of $\pi(T)^{*}-$ $i y I$. Hence the map $\pi(T)^{*}-i y I$ is surjective.

Corollary 6.1. $\quad \hat{\pi}\left(\hat{\mathcal{R}}_{T, y}\right)=\left(y^{2} I+\pi(T)^{*} \overline{\pi(T)}\right)^{-1}$.

Proof. Suppose that $g \in D$ is such that we can find $\ell \geq 0$ for which $g=P_{\ell} \star g=g \star P_{\ell}$. As before, if we define

$$
g_{k}=\left(y^{2} I+A_{k} A_{k}^{*}\right)^{-1} g
$$

then $g_{k}=P_{k} \star g_{k}=g_{k} \star P_{k} \in D$ for all $k \geq \ell$, and

$$
g_{k} \underset{k \rightarrow \infty}{\rightarrow} \hat{\pi}\left(\hat{\mathcal{R}}_{T, y}\right) g
$$

weakly, while

$$
T \star_{1} g_{k} \underset{k \rightarrow \infty}{\rightarrow} \hat{\pi}\left(\mathcal{R}_{T, y} \hat{\star} T\right) g=\hat{\pi}\left(T \hat{\star} \hat{\mathcal{R}}_{T, y}\right) g
$$

in the norm of $H$. Thus $\hat{\pi}\left(\hat{\mathcal{R}}_{T, y}\right) g \in \mathcal{D}(\overline{\pi(T)})$, with

$$
\overline{\pi(T)}\left(\hat{\pi}\left(\hat{\mathcal{R}}_{T, y}\right) g\right)=\hat{\pi}\left(T \hat{\star} \hat{\mathcal{R}}_{T, y}\right) g .
$$

Moreover

$$
\begin{aligned}
& T \hat{\star}\left(\hat{\pi}\left(T \hat{\star} \hat{\mathcal{R}}_{T, y}\right) g\right) \\
& =T \hat{\star}\left[\left(T \hat{\star} \hat{\mathcal{R}}_{T, y}\right) \star_{1} g\right]=\left[T \hat{\star}\left(T \hat{\star} \hat{\mathcal{R}}_{T, y}\right)\right] \star_{1} g \\
& =\left[1-y^{2} \hat{\mathcal{R}}_{T, y}\right] \star_{1} g=g-y^{2} \hat{\pi}\left(\hat{\mathcal{R}}_{T, y}\right) g
\end{aligned}
$$

so we deduce that $\overline{\pi(T)}\left(\hat{\pi}\left(\hat{\mathcal{R}}_{T, y}\right) g\right) \in \mathcal{D}\left(\pi(T)^{*}\right)$ with

$$
\left.\pi(T)^{*}(\overline{\pi(T)})\left(\hat{\pi}\left(\hat{\mathcal{R}}_{T, y}\right) g\right)\right)=g-y^{2} \hat{\pi}\left(\hat{\mathcal{R}}_{T, y}\right) g
$$


This implies that $\hat{\pi}\left(\hat{\mathcal{R}}_{T, y}\right) g$ belongs to $\mathcal{D}\left(\pi(T)^{*} \overline{\pi(T)}\right)$, with

$$
\left(y^{2} I+\pi(T)^{*} \overline{\pi(T)}\right)\left(\hat{\pi}\left(\hat{\mathcal{R}}_{T, y}\right) g\right)=g
$$

which implies that $\hat{\pi}\left(\hat{\mathcal{R}}_{T, y}\right) g=\left(y^{2} I+\pi(T)^{*} \overline{\pi(T)}\right)^{-1} g$.

The proper description of the distribution $\mathcal{R}_{T, y}$ is not so straightforward. There is an interesting relationship between $\mathcal{R}_{T, y}$ and $\hat{\mathcal{R}}_{T, y}$, and the 'amount by which $\mathcal{R}_{T, y}$ differs from $\hat{\mathcal{R}}_{T, y}$ ' turns out, in effect, to be the 'amount by which $\pi(T)$ fails to be essentially self-adjoint'. To establish this result requires another technical lemma.

Lemma 6.5. The distribution $\mathcal{R}_{T, y}$ satisfies the following:

1. $0 \leq \hat{\mathcal{R}}_{T, y} \leq \mathcal{R}_{T, y}$ as elements of the $C^{*}$-algebra $D_{b}^{\prime}$,

2. $y^{2}\left\{\mathcal{R}_{T, y} \hat{\star}\left(T \hat{\star} \mathcal{R}_{T, y}\right)-\left(\mathcal{R}_{T, y} \hat{\star} T\right) \hat{\star} \mathcal{R}_{T, y}\right\}=T \hat{\star} \mathcal{R}_{T, y}-\mathcal{R}_{T, y} \hat{\star} T$.

Proof. Since

$$
\begin{aligned}
{\left[T \hat{\star} \mathcal{R}_{T, y}\right] \hat{\star}\left[T \hat{\star} \mathcal{R}_{T, y}\right] } & =\left[\hat{\mathcal{R}}_{T, y} \hat{\star} T\right] \hat{\star}\left[T \hat{\star} \mathcal{R}_{T, y}\right]=\hat{\mathcal{R}}_{T, y} \hat{\star}\left[T \hat{\star}\left(T \hat{\star} \mathcal{R}_{T, y}\right)\right] \\
& =\hat{\mathcal{R}}_{T, y}-y^{2} \hat{\mathcal{R}}_{T, y} \hat{\star} \mathcal{R}_{T, y} \\
{\left[T \hat{\star} \mathcal{R}_{T, y}\right] \hat{\star}\left[T \hat{\star} \hat{\mathcal{R}}_{T, y}\right] } & =\left[\left(T \hat{\star} \mathcal{R}_{T, y}\right) \hat{\star} T\right] \hat{\star} \hat{\mathcal{R}}_{T, y}=\hat{\mathcal{R}}_{T, y}-y^{2} \hat{\mathcal{R}}_{T, y} \hat{\star} \hat{\mathcal{R}}_{T, y}
\end{aligned}
$$

we deduce that

$$
\left[T \hat{\star} \mathcal{R}_{T, y}\right] \hat{\star}\left\{T \hat{\star}\left(\mathcal{R}_{T, y}-\hat{\mathcal{R}}_{T, y}\right)\right\}=-y^{2} \hat{\mathcal{R}}_{T, y} \hat{\star}\left[\mathcal{R}_{T, y}-\hat{\mathcal{R}}_{T, y}\right]
$$

Similarly

$$
\begin{aligned}
{\left[T \hat{\star} \hat{\mathcal{R}}_{T, y}\right] \hat{\star}\left[T \hat{\star} \mathcal{R}_{T, y}\right] } & =\left[\mathcal{R}_{T, y} \hat{\star} T\right] \hat{\star}\left[\hat{\mathcal{R}}_{T, y} \hat{\star} T\right]=\left[\left(\mathcal{R}_{T, y} \hat{\star} T\right) \hat{\star} \hat{\mathcal{R}}_{T, y}\right] \hat{\star} T \\
& =\left[\mathcal{R}_{T, y} \hat{\star}\left(T \hat{\star} \hat{\mathcal{R}}_{T, y}\right)\right] \hat{\star} T=\mathcal{R}_{T, y} \hat{\star}\left[\left(T \hat{\star} \hat{\mathcal{R}}_{T, y}\right) \hat{\star} T\right] \\
& =\mathcal{R}_{T, y}-y^{2} \mathcal{R}_{T, y} \hat{\star} \mathcal{R}_{T, y} \\
{\left[T \hat{\star} \hat{\mathcal{R}}_{T, y}\right] \hat{\star}\left[T \hat{\star} \hat{\mathcal{R}}_{T, y}\right] } & =\left[\left(T \hat{\star} \hat{\mathcal{R}}_{T, y}\right) \hat{\star} T\right] \hat{\star} \hat{\mathcal{R}}_{T, y}=\hat{\mathcal{R}}_{T, y}-y \mathcal{R}_{T, y} \hat{\star} \hat{\mathcal{R}}_{T, y}
\end{aligned}
$$

so that

$$
\left[T \hat{\star} \hat{\mathcal{R}}_{T, y}\right] \hat{\star}\left\{T \hat{\star}\left(\mathcal{R}_{T, y}-\hat{\mathcal{R}}_{T, y}\right)\right\}=\left[\mathcal{R}_{T, y}-\hat{\mathcal{R}}_{T, y}\right]-y^{2} \mathcal{R}_{T, y} \hat{\star}\left[\mathcal{R}_{T, y}-\hat{\mathcal{R}}_{T, y}\right]
$$

Thus

$$
\begin{aligned}
\mathcal{R}_{T, y}-\hat{\mathcal{R}}_{T, y}= & y^{2}\left[\mathcal{R}_{T, y}-\hat{\mathcal{R}}_{T, y}\right] \hat{\star}\left[\mathcal{R}_{T, y}-\hat{\mathcal{R}}_{T, y}\right] \\
& -\left\{T \hat{\star}\left[\mathcal{R}_{T, y}-\hat{\mathcal{R}}_{T, y}\right] \hat{\star}\left\{T \hat{\star}\left[\mathcal{R}_{T, y}-\hat{\mathcal{R}}_{T, y}\right]\right\}\right. \\
= & y^{2}\left[\mathcal{R}_{T, y}-\hat{\mathcal{R}}_{T, y}\right] \hat{\star}\left[\mathcal{R}_{T, y}-\hat{\mathcal{R}}_{T, y}\right] \\
& +\overline{\left\{T \hat{\star}\left[\mathcal{R}_{T, y}-\hat{\mathcal{R}}_{T, y}\right]\right\}} \hat{\star}\left\{T \hat{\star}\left[\mathcal{R}_{T, y}-\hat{\mathcal{R}}_{T, y}\right]\right\} \\
\geq & 0
\end{aligned}
$$


which shows that $0 \leq \hat{\mathcal{R}}_{T, y} \leq \mathcal{R}_{T, y}$. Moreover, since

$$
\begin{aligned}
& {\left[1-y^{2} \mathcal{R}_{T, y}\right] \hat{\star}\left(T \hat{\star} \mathcal{R}_{T, y}\right)} \\
& =\left[T \hat{\star}\left(T \hat{\star} \mathcal{R}_{T, y}\right)\right] \hat{\star}\left(T \hat{\star} \mathcal{R}_{T, y}\right)=T \hat{\star}\left[\left(T \hat{\star} \mathcal{R}_{T, y}\right) \hat{\star}\left(T \hat{\star} \mathcal{R}_{T, y}\right)\right] \\
& =T \hat{\star}\left(\hat{\mathcal{R}}_{T, y}-y^{2} \hat{\mathcal{R}}_{T, y} \hat{\star} \mathcal{R}_{T, y}\right)=T \hat{\star} \hat{\mathcal{R}}_{T, y}-y^{2} T \hat{\star}\left(\hat{\mathcal{R}}_{T, y} \hat{\star} \mathcal{R}_{T, y}\right) \\
& =T \hat{\star} \hat{\mathcal{R}}_{T, y}-y^{2}\left(T \hat{\star} \hat{\mathcal{R}}_{T, y}\right) \hat{\star} \mathcal{R}_{T, y}=T \hat{\star} \hat{\mathcal{R}}_{T, y}-y^{2}\left(\mathcal{R}_{T, y} \hat{\star} T\right) \hat{\star} \mathcal{R}_{T, y}
\end{aligned}
$$

we deduce that

$$
\begin{aligned}
y^{2}\left\{\mathcal{R}_{T, y} \hat{\star}\left(T \hat{\star} \mathcal{R}_{T, y}\right)-\left(\mathcal{R}_{T, y} \hat{\star} T\right) \hat{\star} \mathcal{R}_{T, y}\right\} & =T \hat{\star}\left[\mathcal{R}_{T, y}-\hat{\mathcal{R}}_{T, y}\right] \\
& =T \hat{\star} \mathcal{R}_{T, y}-\mathcal{R}_{T, y} \hat{\star} T
\end{aligned}
$$

We now have enough machinery to introduce the following important distribution. Define

$$
\begin{aligned}
\mathcal{E}_{T, y} & =y^{2}\left[\mathcal{R}_{T, y}-\hat{\mathcal{R}}_{T, y}\right]+i y T \hat{\star}\left[\mathcal{R}_{T, y}-\hat{\mathcal{R}}_{T, y}\right] \\
& =y^{2}\left[\mathcal{R}_{T, y}-\hat{\mathcal{R}}_{T, y}\right]-i y\left[\mathcal{R}_{T, y}-\hat{\mathcal{R}}_{T, y}\right] \hat{\star} T \\
& =-i y\left[\mathcal{R}_{T, y}-\hat{\mathcal{R}}_{T, y}\right] \hat{\star}(T+i y) \\
& =-i y\left[\mathcal{S}_{T, y}-\hat{\mathcal{S}}_{T, y}\right]
\end{aligned}
$$

Proposition 6.6. We have $\varepsilon_{T, y}=\overline{\varepsilon_{T, y}}=\varepsilon_{T, y} \hat{\star} \varepsilon_{T, y}$. Moreover, $\mathcal{E}_{T, y} \in D_{b}^{\prime}$, and has the positivity property $0 \leq \mathcal{E}_{T, y} \leq 1$, and $(T+i y) \hat{\star} \mathcal{E}_{T, y}=$ $\mathcal{E}_{T, y} \hat{\star}(T-i y)=0$.

Proof. The first of the two equivalent expressions for $\varepsilon_{T, y}$ show clearly that $\overline{\mathcal{E}_{T, y}}=\mathcal{E}_{T, y}$. Since

$$
\begin{aligned}
\mathcal{E}_{T, y} \hat{\star} \mathcal{E}_{T, y} & \\
= & y^{4}\left[\mathcal{R}_{T, y}-\hat{\mathcal{R}}_{T, y}\right] \hat{\star}\left[\mathcal{R}_{T, y}-\hat{\mathcal{R}}_{T, y}\right]-y^{2}\left(T \hat{\star}\left[\mathcal{R}_{T, y}-\hat{\mathcal{R}}_{T, y}\right]\right) \hat{\star}\left(T \hat{\star}\left[\mathcal{R}_{T, y}-\hat{\mathcal{R}}_{T, y}\right]\right) \\
& +i y^{3}\left\{\left[\mathcal{R}_{T, y}-\hat{\mathcal{R}}_{T, y}\right] \hat{\star}\left(T \hat{\star}\left[\mathcal{R}_{T, y}-\hat{\mathcal{R}}_{T, y}\right]\right)+\left(T \hat{\star}\left[\mathcal{R}_{T, y}-\hat{\mathcal{R}}_{T, y}\right]\right) \hat{\star}\left[\mathcal{R}_{T, y}-\hat{\mathcal{R}}_{T, y}\right]\right\} \\
= & y^{2}\left[\mathcal{R}_{T, y}-\hat{\mathcal{R}}_{T, y}\right]+i y^{3}\left\{\mathcal{R}_{T, y} \hat{\star}\left(T \star \hat{\star} \mathcal{R}_{T, y}\right)-\left(\mathcal{R}_{T, y} \hat{\star} T\right) \hat{\star} \mathcal{R}_{T, y}\right\} \\
= & y^{2}\left[\mathcal{R}_{T, y}-\hat{\mathcal{R}}_{T, y}\right]+i y T \hat{\star}\left[\mathcal{R}_{T, y}-\hat{\mathcal{R}}_{T, y}\right]=\mathcal{E}_{T, y}
\end{aligned}
$$

we deduce that $\mathcal{E}_{T, y}$ is also idempotent. Hence $\mathcal{E}_{T, y}$ is a self-adjoint idempotent in the $C^{*}$-algebra $D_{b}^{\prime}$, so $0 \leq \mathcal{E}_{T, y} \leq 1$ follows. It is clear that

$$
\mathcal{E}_{T, y} \hat{\star}(T-i y 1)=-i y\left[\mathcal{S}_{T, y}-\hat{\mathcal{S}}_{T, y}\right] \hat{\star}(T-i y 1)=-i y[1-1]=0
$$


and we deduce that $(T+i y 1) \hat{\star} \mathcal{E}_{T, y}=\overline{\mathcal{E}_{T, y} \hat{\star}(T-i y 1)}=0$ as well.

We observed previously that $\mathcal{S}_{T, y}$ and $\hat{\mathcal{S}}_{T, y}$ were one-sided inverses for $T-i y 1$. This new distribution $\mathcal{E}_{T, y}$ measures the amount by which they are not two-sided inverses.

Corollary 6.2. We have the identities

$$
(T-i y 1) \hat{\star} \mathcal{S}_{T, y}=1+\mathcal{E}_{T, y}, \quad(T-i y 1) \hat{\star} \hat{\mathcal{S}}_{T, y}=1-\mathcal{E}_{T, y}
$$

Proof. We have

$$
\begin{aligned}
(T-i y 1) \hat{\star} \mathcal{S}_{T, y} & =(T-i y 1) \hat{\star}\left(\mathcal{R}_{T, y} \hat{\star} T+i y \mathcal{R}_{T, y}\right) \\
& =T \hat{\star}\left(\mathcal{R}_{T, y} \hat{\star} T\right)-i y \mathcal{R}_{T, y} \hat{\star} T+i y T \hat{\star} \mathcal{R}_{T, y}+y^{2} \mathcal{R}_{T, y} \\
& =1+y^{2}\left[\mathcal{R}_{T, y}-\hat{\mathcal{R}}_{T, y}\right]+i y T \hat{\star}\left[\mathcal{R}_{T, y}-\hat{\mathcal{R}}_{T, y}\right] \\
& =1+\mathcal{E}_{T, y}
\end{aligned}
$$

Since $(T-i y 1) \hat{\star} \mathcal{Z}_{T, y}=1$, the other equation is immediate.

We can now characterize the distribution $\mathcal{E}_{T, y}$ in terms of one of the deficiency spaces for $\pi(T)^{*}$.

Proposition 6.7. $\hat{\pi}\left(\mathcal{E}_{T, y}\right) \in \mathcal{B}_{\star}(H)$ is the orthogonal projection onto the deficiency space $\operatorname{Ker}\left(\pi(T)^{*}+i y I\right)$.

Proof. If $\phi \in H$ then $\mathcal{E}_{T, y} \hat{\star} \phi \in H$ as well and

$$
(T+i y 1) \hat{\star}\left(\mathcal{E}_{T, y} \hat{\star} \phi\right)=\left[(T+i y 1) \hat{\star} \mathcal{E}_{T, y}\right] \hat{\star} \phi=0 \hat{\star} \phi=0
$$

so $T \hat{\star}\left(\mathcal{E}_{T, y} \hat{\star} \phi\right)=-i y \mathcal{E}_{T, y} \hat{\star} \phi$ belongs to $H$. Thus $\mathcal{E}_{T, y} \hat{\star} \phi \in \mathcal{D}\left(\pi(T)^{*}\right)$, with

$$
\left(\pi(T)^{*}+i y I\right)\left(\varepsilon_{T, y} \hat{\star} \phi\right)=0 .
$$

Thus we deduce that $\hat{\pi}\left(\varepsilon_{T, y}\right) \phi=\varepsilon_{T, y} \hat{\star} \phi$ belongs to the deficiency space $\operatorname{Ker}\left(\pi(T)^{*}+i y I\right)$.

Conversely, if $\phi \in \operatorname{Ker}\left(\pi(T)^{*}+i y I\right)$ then $(T+i y 1) \hat{\star} \phi=0$, so

$$
\hat{\mathcal{S}}_{T, y} \hat{\star} \phi=\left[\hat{\mathcal{R}}_{T, y} \hat{\star}(T+i y 1)\right] \hat{\star} \phi=\hat{\mathcal{R}}_{T, y} \hat{\star}[(T+i y 1) \hat{\star} \phi]=0
$$

and hence

$$
\begin{aligned}
\phi-\mathcal{E}_{T, y} \hat{\star} \phi & =\left(1-\mathcal{E}_{T, y}\right) \hat{\star} \phi=\left[(T-i y 1) \hat{\star} \hat{\mathcal{S}}_{T, y}\right] \hat{\star} \phi \\
& =(T-i y 1) \hat{\star}\left[\hat{\mathcal{S}}_{T, y} \hat{\star} \phi\right]=0
\end{aligned}
$$

which tells us that $\hat{\pi}\left(\mathcal{E}_{T, y}\right) \phi=\mathcal{E}_{T, y} \hat{\star} \phi=\phi$.

We can now describe the distribution $\mathcal{R}_{T, y}$ exactly in terms of $\pi(T)$. 


\section{Corollary 6.3. $\quad$ Since}

$$
\mathcal{R}_{T, y}=\hat{\mathcal{R}}_{T, y}+\frac{1}{2 y^{2}}\left\{\mathcal{E}_{T, y}+\mathcal{E}_{T,-y}\right\}
$$

we see that the operator $\hat{\pi}\left(\mathcal{R}_{T, y}\right) \in \mathcal{B}_{\star}(H)$ can be described as a linear combination of $\left(y^{2} I+\pi(T)^{*} \overline{\pi(T)}\right)^{-1}$ and the orthogonal projections onto the two deficiency subspaces $\operatorname{Ker}\left(\pi(T)^{*}+i y I\right)$ and $\operatorname{Ker}\left(\pi(T)^{*}-i y I\right)$.

A related spectral result we can prove at this point is the identification of the Cayley transform of $\pi(T)$. The next result represents an extension of the arguments used in Corollary 6.1.

Lemma 6.6. If $\phi \in H$ then $\hat{\pi}\left(\hat{\mathcal{S}}_{T,-1}\right) \phi \in \mathcal{D}(\overline{\pi(T)})$.

Proof. For any $k \in \mathbb{N}$ define

$$
\begin{aligned}
\alpha_{k} & =\left(I+A_{k}^{*} A_{k}\right)^{-1}\left(A_{k}^{*}-i I\right) \phi \\
\beta_{k} & =\left(I+A_{k} A_{k}^{*}\right)^{-1}\left(A_{k}-i I\right) \phi \\
\gamma_{k} & =\left(\left(I+A_{k} A_{k}^{*}\right)^{-1}\left(A_{k}-i \Pi_{k}\right) \phi\right) \star P_{k}=P_{k} \star \beta_{k} \star P_{k}
\end{aligned}
$$

noting that $\left\|\alpha_{k}\right\|,\left\|\beta_{k}\right\| \leq 2\|\phi\|$ for all $k \in \mathbb{N}$. Clearly $\gamma_{k} \in D$ for all $k \in \mathbb{N}$, and since

$$
\begin{aligned}
\left|\left(\psi \mid \hat{\pi}\left(\hat{\mathcal{S}}_{T,-1}\right) \phi-\gamma_{k}\right)_{2}\right| & \leq\left|\left(\psi \mid \hat{\pi}\left(\hat{\mathcal{S}}_{T,-1}\right) \phi-\beta_{k}\right)_{2}\right|+\left|\left(\psi \mid \beta_{k}-P_{k} \star \beta_{k} \star P_{k}\right)_{2}\right| \\
& =\left|\left(\psi \mid \hat{\pi}\left(\hat{\mathcal{S}}_{T,-1}\right) \phi-\beta_{k}\right)_{2}\right|+\left|\left(\psi-P_{k} \star \psi \star P_{k} \mid \beta_{k}\right)_{2}\right| \\
& \leq\left|\left(\psi \mid \hat{\pi}\left(\hat{\mathcal{S}}_{T,-1}\right) \phi-\beta_{k}\right)_{2}\right|+2\|\phi\|_{2} \cdot\left\|\psi-P_{k} \star \psi \star P_{k}\right\|_{2}
\end{aligned}
$$

for any $\psi \in H$, and since the sequence $\left(\beta_{k}\right)_{k}$ converges to $\hat{\pi}\left(\hat{\mathcal{S}}_{T,-1}\right) \phi$ weakly, we deduce that $\left(\gamma_{k}\right)_{k}$ converges to $\hat{\pi}\left(\hat{\mathcal{S}}_{T,-1}\right) \phi$ weakly.

Moreover

$$
\begin{aligned}
\pi(T) \gamma_{k} & =\left(A_{k}^{*} \beta_{k}\right) \star P_{k}=\left(\left(I+A_{k}^{*} A_{k}\right)^{-1} A_{k}^{*}\left(A_{k}-i I\right) \phi\right) \star P_{k} \\
& =\left(\phi-\left(I+A_{k}^{*} A_{k}\right)^{-1}\left(I+i A_{k}^{*}\right) \phi\right) \star P_{k}=\left(\phi-i \alpha_{k}\right) \star P_{k}
\end{aligned}
$$

so we see that

$$
\begin{aligned}
& \left\|\pi(T) \gamma_{k}-\phi+i \hat{\pi}\left(\mathcal{S}_{T,-1}\right) \phi\right\|_{2} \\
& \leq\left\|\phi-\phi \star P_{k}\right\|_{2}+\left\|\hat{\pi}\left(\mathcal{S}_{T,-1}\right) \phi-\left(\hat{\pi}\left(\mathcal{S}_{T,-1}\right) \phi\right) \star P_{k}\right\|_{2}+\left\|\left(\hat{\pi}\left(\mathcal{S}_{T,-1}\right) \phi-\alpha_{k}\right) \star P_{k}\right\|_{2} \\
& \leq\left\|\phi-\phi \star P_{k}\right\|_{2}+\left\|\hat{\pi}\left(\mathcal{S}_{T,-1}\right) \phi-\left(\hat{\pi}\left(\mathcal{S}_{T,-1}\right) \phi\right) \star P_{k}\right\|_{2}+\left\|\hat{\pi}\left(\mathcal{S}_{T,-1}\right) \phi-\alpha_{k}\right\|_{2}
\end{aligned}
$$


so that fact that $\left(\alpha_{k}\right)_{k}$ converges strongly to $\hat{\pi}\left(\mathcal{S}_{T,-1}\right) \phi$ tells us that $\left(\pi(T) \gamma_{k}\right)_{k}$ converges strongly to $\phi-i \hat{\pi}\left(\mathcal{S}_{T,-1}\right) \phi$. Thus we deduce that $\hat{\pi}\left(\hat{\mathcal{S}}_{T,-1}\right) \phi$ belongs to the domain $\mathcal{D}(\overline{\pi(T)})$, as required, and also that

$$
\overline{\pi(T)} \hat{\pi}\left(\hat{\mathcal{S}}_{T,-1}\right) \phi=\phi-i \hat{\pi}\left(\mathcal{S}_{T,-1}\right) \phi
$$

Consider the distribution $U \in D_{b}^{\prime}$ defined by the formula

$$
U=(T-i 1) \hat{\star} \hat{\mathcal{S}}_{T,-1}=(T-i 1) \hat{\star}\left(\hat{\mathcal{R}}_{T, 1} \hat{\star}(T-i 1)\right)
$$

Proposition 6.8. The operator $\hat{\pi}(U)$ is equal to the Cayley transform $\mathcal{U}$ of the closed symmetric operator $\overline{\pi(T)}$.

Proof. If $\phi \in \operatorname{Ker}\left(\pi(T)^{*}-i I\right)$ then

$$
\begin{aligned}
\hat{\mathcal{S}}_{T,-1} \hat{\star} \phi & =\left(\hat{\mathcal{R}}_{T,-1} \hat{\star}(T-i 1)\right) \hat{\star} \phi=\hat{\mathcal{R}}_{T,-1} \hat{\star}((T-i 1) \hat{\star} \phi) \\
& =\hat{\mathcal{R}}_{T,-1} \hat{\star}\left(\left(\pi(T)^{*}-i I\right) \phi\right)=0
\end{aligned}
$$

and hence

$$
U \hat{\star} \phi=(T-i 1) \hat{\star}\left(\hat{\mathfrak{S}}_{T,-1} \hat{\star} \phi\right)=0=\mathcal{U} \phi
$$

On the other hand, if $\phi \in \operatorname{Ker}\left(\pi(T)^{*}-i I\right)^{\perp}$ then, since $\hat{\pi}\left(\mathcal{E}_{T,-1}\right)$ is the orthogonal projection onto $\operatorname{Ker}\left(\pi(T)^{*}-i I\right)$, it follows that

$$
\begin{aligned}
\phi & =\left(I-\hat{\pi}\left(\mathcal{E}_{T,-1}\right)\right) \phi=\left(1-\mathcal{E}_{T,-1}\right) \hat{\star} \phi \\
& =\left[(T+i 1) \hat{\star} \hat{\mathcal{S}}_{T,-1}\right] \hat{\star} \phi=(T+i 1) \hat{\star}\left[\hat{\mathcal{S}}_{T,-1} \hat{\star} \phi\right] \\
& =\left(\hat{\pi}(T)^{*}+i I\right)\left[\hat{\mathcal{S}}_{T,-1} \hat{\star} \phi\right]
\end{aligned}
$$

and so, in fact, $\hat{\mathcal{S}}_{T,-1} \hat{\star} \phi$ is that element $\psi$ of $\mathcal{D}(\overline{\pi(T)})$ for which $\phi=(\overline{\pi(T)}+$ $i I) \psi$. But then

$$
\begin{aligned}
U \hat{\star} \phi & =(T-i 1) \hat{\star}\left(\hat{\mathcal{S}}_{T,-1} \hat{\star} \phi\right)=(T-i 1) \hat{\star} \psi \\
& =\left(\pi(T)^{*}-i I\right) \psi=(\overline{\pi(T)}-i I) \psi \\
& =\mathcal{U} \phi
\end{aligned}
$$

Thus we have shown that $U \hat{\star} \phi=\mathcal{U} \phi$ for all $\phi \in H$, as required.

We end this section with a theorem which establishes a series of necessary and sufficient conditions for the operator $\pi(T)$ to be essentially self-adjoint. It is important to note that [9] contains a (small) number of errors, effectively assuming identities (3) and (4), which means that the later results of spectral theory are not surprising, since they are simply those results that can be achieved, as ever, for essentially self-adjoint operators. 
Theorem 6.1. The following are equivalent:

1. The operator $\pi(T): D \rightarrow H$ is essentially self-adjoint,

2. $\mathcal{R}_{T, y}=\hat{\mathcal{R}}_{T, y}$,

3. $T \hat{\star} \mathcal{R}_{T, y}=\mathcal{R}_{T, y} \hat{\star} T$,

4. $\mathcal{R}_{T, y} \hat{\star}\left(T \hat{\star} \mathcal{R}_{T, y}\right)=\left(\mathcal{R}_{T, y} \hat{\star} T\right) \hat{\star} \mathcal{R}_{T, y}$,

5. $y^{2}\left[\mathcal{R}_{T, y}-\hat{\mathcal{R}}_{T, y}\right]$ is idempotent,

6. $\mathcal{E}_{T, y}=\mathcal{E}_{T,-y}=0$.

Proof.

$(2) \Rightarrow(3)$ : If $\mathcal{R}_{T, y}=\hat{\mathcal{R}}_{T, y}$, then $T \hat{\star} \mathcal{R}_{T, y}=\hat{\mathcal{R}}_{T, y} \hat{\star} T=\mathcal{R}_{T, y} \hat{\star} T$.

$(3) \Rightarrow(2)$ : If $T \hat{\star} \mathcal{R}_{T, y}=\mathcal{R}_{T, y} \hat{\star} T$, then

$$
1-y^{2} \mathcal{R}_{T, y}=\left(\mathcal{R}_{T, y} \hat{\star} T\right) \hat{\star} T=\left(T \hat{\star} \mathcal{R}_{T, y}\right) \hat{\star} T=1-y^{2} \hat{\mathcal{R}}_{T, y}
$$
and hence $\mathcal{R}_{T, y}=\hat{\mathcal{R}}_{T, y}$.

$(3) \Leftrightarrow(4)$ : See Lemma 6.5 .

$(3) \Leftrightarrow(5)$ : Writing $P=y^{2}\left[\mathcal{R}_{T, y}-\hat{\mathcal{R}}_{T, y}\right]$, we showed in the proof of Lemma 6.5 that

$$
P-P^{2}=y^{2} \overline{\left[T \hat{\star}\left(\mathcal{R}_{T, y}-\hat{\mathcal{R}}_{T, y}\right)\right]} \hat{\star}\left[T \hat{\star}\left(\mathcal{R}_{T, y}-\hat{\mathcal{R}}_{T, y}\right)\right]
$$

and hence (5) is equivalent to the statement that

$$
\overline{\left[T \hat{\star}\left(\mathcal{R}_{T, y}-\hat{\mathcal{R}}_{T, y}\right)\right]} \hat{\star}\left[T \hat{\star}\left(\mathcal{R}_{T, y}-\hat{\mathcal{R}}_{T, y}\right)\right]=0
$$

which is equivalent to $T \hat{\star}\left(\mathcal{R}_{T, y}-\hat{\mathcal{R}}_{T, y}\right)=0$, which is equivalent to $(3)$.

$(2) \Rightarrow(6):$ If $\mathcal{R}_{T, y}=\hat{\mathcal{R}}_{T, y}$, then

$$
\mathcal{E}_{T, \pm y}=\left(y^{2} 1 \pm i y T\right) \hat{\star}\left(\mathcal{R}_{T, y}-\hat{\mathcal{R}}_{T, y}\right)=0
$$

$(6) \Rightarrow(2)$ : If $\mathcal{E}_{T, y}=\mathcal{E}_{T,-y}=0$ then

$$
2 y^{2}\left[\mathcal{R}_{T, y}-\hat{\mathcal{R}}_{T, y}\right]=\mathcal{E}_{T, y}+\mathcal{E}_{T,-y}=0
$$

and hence $\mathcal{R}_{T, y}=\hat{\mathcal{R}}_{T, y}$.

$(1) \Leftrightarrow(6)$ : This is standard, given our characterization of $\mathcal{E}_{T, y}$ and $\mathcal{E}_{T,-y}$ in terms of the deficiency subspaces. 


\section{§7. Aiming for a Spectral Calculus}

We have shown that some of the key results in [9] are suspect, except in the simple case that $\pi(T)$ is essentially self-adjoint. The root problem would seem to be Theorem 7.5 of that paper, which introduces $\left(1+T^{2}\right)^{-1}$, and claims that it commutes with $T$. As we have seen, this statement fails to distinguish between $\mathcal{R}_{T, 1}$ and $\hat{\mathcal{R}}_{T, 1}$.

The question remains whether it is still possible to retrieve some of the results in that paper by constructing a spectral calculus, even when $\pi(T)$ is not essentially self-adjoint. To do this, we need to be able to create a distributionvalued resolvent function which has "good" properties, analyticity for example. Unfortunately, we can show that this is not possible, at least with the distributions we have identified so far.

Let us continue to work with a fixed "real" distribution $T=\bar{T} \in Q \cap \bar{Q}$. For any complex number $z \in \mathbb{C} \backslash \mathbb{R}$ (so that we can write $z=x+i y$ where $x, y \in \mathbb{R}$ and $y$ is nonzero), we shall introduce the notation:

$$
\begin{array}{lll}
\mathcal{R}_{z}=\mathcal{R}_{T-x 1, y} & \mathcal{S}_{z}=\mathcal{S}_{T-x 1, y} & \mathcal{E}_{z}=\mathcal{E}_{T-x 1, y} \\
\hat{\mathcal{R}}_{z}=\hat{\mathcal{R}}_{T-x 1, y} & \hat{\mathcal{S}}_{z}=\hat{\mathcal{S}}_{T-x 1, y} & \mathcal{Z}_{z}=\mathcal{Z}_{T-x 1, y}
\end{array}
$$

Using this notation, we can summarize some of the key results of previous sections as follows:

- $\mathcal{S}_{z}=\mathcal{R}_{z} \hat{\star}(T-\bar{z} 1)$ and $\hat{\mathcal{S}}_{z}=\hat{\mathcal{R}}_{z} \hat{\star}(T-\bar{z} 1)$,

- $\mathcal{S}_{z} \hat{\star}(T-z 1)=\hat{\mathcal{S}}_{z} \hat{\star}(T-z 1)=1$,

- $(T-z 1) \hat{\star} \mathcal{S}_{z}=1+\mathcal{E}_{z}$, and $(T-z 1) \hat{\star} \hat{\mathcal{S}}_{z}=1-\mathcal{E}_{z}$,

- $z_{z} \hat{\star}(T-z 1)=(T-z 1) \hat{\star} z_{z}=1$, while $\overline{z_{z}}=z_{\bar{z}}$,

- All of these distributions belong to $D_{b}^{\prime}$, with

$$
\left\|\mathcal{R}_{z}\right\|,\left\|\hat{\mathcal{R}}_{z}\right\| \leq \frac{1}{(\operatorname{Im} z)^{2}} \quad\left\|\mathcal{S}_{z}\right\|,\left\|\hat{\mathcal{S}}_{z}\right\|,\left\|z_{z}\right\| \leq \frac{1}{|\operatorname{Im} z|} \quad\left\|\mathcal{E}_{z}\right\| \leq 1
$$

- $\mathcal{E}_{z}=\overline{\mathcal{E}_{z}}=\mathcal{E}_{z} \hat{\star} \mathcal{E}_{z}$, and $\hat{\pi}\left(\mathcal{E}_{z}\right)$ is the orthogonal projection onto the space $\operatorname{Ker}\left(\pi(T)^{*}-\bar{z} I\right)$.

In order to implement some form of spectral calculus, we will need some variant of the usual resolvent formula, since the latter does not hold here. We shall show some of the formulae that are possible in this general framework. 
We already have three candidate resolvent functions: $\mathcal{S}, \hat{\mathcal{S}}$ and $\mathcal{Z}$. Of these, the last is the most attractive since it is a full-blooded two-sided resolvent function, while the other two are only one-sided.

The following technical lemmata are crucial in what follows:

Lemma 7.1. For any $z, w \in \mathbb{C} \backslash \mathbb{R}$, we have the following identities:

1. $\hat{\mathcal{S}}_{w} \hat{\star} \mathcal{E}_{z}=(\bar{z}-\bar{w}) \hat{\mathcal{R}}_{w} \hat{\star} \mathcal{E}_{z}$.

2. $\mathcal{S}_{z} \hat{\star} \mathcal{E}_{z}=\frac{i}{\operatorname{Im} z} \varepsilon_{z}$

3. $z_{z} \hat{\star} \mathcal{E}_{z}=\frac{i}{2 \operatorname{Im} z} \varepsilon_{z}$

Proof. We first note that

$\hat{\mathcal{S}}_{w} \hat{\star} \mathcal{E}_{z}=\left[\hat{\mathcal{R}}_{w} \hat{\star}(T-\bar{w} 1)\right] \hat{\star} \mathcal{E}_{z}=\hat{\mathcal{R}}_{w} \hat{\star}\left[(T-\bar{w} 1) \hat{\star} \mathcal{E}_{z}\right]=(\bar{z}-\bar{w}) \hat{\mathcal{R}}_{w} \hat{\star} \mathcal{E}_{z}$ and so, in particular, $\hat{\mathcal{S}}_{z} \hat{\star} \mathcal{E}_{z}=0$. Since $\mathcal{S}_{z}=\hat{\mathcal{S}}_{z}+\frac{i}{\operatorname{Im} z} \mathcal{E}_{z}$, we deduce that

$$
\mathcal{S}_{z} \hat{\star} \mathcal{E}_{z}=\hat{\mathcal{S}}_{z} \hat{\star} \mathcal{E}_{z}+\frac{i}{\operatorname{Im} z} \mathcal{E}_{z} \hat{\star} \mathcal{E}_{z}=\frac{i}{\operatorname{Im} z} \mathcal{E}_{z}
$$

as required. The last result follows since $z_{z}=\frac{1}{2}\left[\mathcal{S}_{z}+\hat{\mathcal{S}}_{z}\right]$.

Lemma 7.2. If $X \in D_{b}^{\prime}$ is such that $X \hat{\star}(T-z 1)=0$, then $X=$ $X \hat{\star} \mathcal{E}_{z}$.

Proof. We have

$$
\begin{aligned}
& X-X \hat{\star} \mathcal{E}_{z} \\
& =X \hat{\star}\left[1-\mathcal{E}_{z}\right]=X \hat{\star}\left[(T-z 1) \hat{\star} \hat{\mathcal{S}}_{z}\right] \\
& =X \hat{\star}\left[(T-z 1) \hat{\star}\left(\hat{\mathcal{R}}_{z} \hat{\star}(T-\bar{z} 1)\right)\right]=X \hat{\star}\left[\left((T-z 1) \hat{\star} \hat{\mathcal{R}}_{z}\right) \hat{\star}(T-\bar{z} 1)\right] \\
& =\left[X \hat{\star}\left((T-z 1) \hat{\star} \hat{\mathcal{R}}_{z}\right)\right] \hat{\star}(T-\bar{z} 1)=\left[(X \hat{\star}(T-z 1)) \hat{\star} \hat{\mathcal{R}}_{z}\right] \hat{\star}(T-\bar{z} 1) \\
& =\left[0 \hat{\star} \hat{\mathcal{R}}_{z}\right] \hat{\star}(T-\bar{z} 1)=0
\end{aligned}
$$

carefully using the available results on associativity of the product $\hat{\star}$.

Putting these results together, we deduce that there is a further interesting relationship between these resolvent functions. 
Proposition 7.1. For any $z \in \mathbb{C} \backslash \mathbb{R}$ we have

$$
\mathcal{S}_{z}=z_{z}+\frac{i}{2 \operatorname{Im} z} \varepsilon_{z} \quad \hat{\mathcal{S}}_{z}=z_{z}-\frac{i}{2 \operatorname{Im} z} \varepsilon_{z}
$$

Proof. Certainly $\left(\mathcal{Z}_{z}-\hat{\mathcal{S}}_{z}\right) \hat{\star}(T-z 1)=1-1=0$, and hence we deduce that

$$
z_{z}-\hat{\mathcal{S}}_{z}=\left(z_{z}-\hat{\mathcal{S}}_{z}\right) \hat{\star} \mathcal{E}_{z}=z_{z} \hat{\star} \mathcal{E}_{z}=\frac{i}{2 \operatorname{Im} z} \varepsilon_{z}
$$

as required. The second identity follows since $z_{z}=\frac{1}{2}\left[\mathcal{S}_{z}+\hat{\mathcal{S}}_{z}\right]$.

We can now establish the nearest thing that we have to the resolvent formula for $z$

Theorem 7.1. For any $z, w \in \mathbb{C} \backslash \mathbb{R}$, we have

$$
z_{w}-z_{z}=(w-z) z_{w} \hat{\star} z_{z}+\frac{\bar{w}-\bar{z}}{4 \operatorname{Im} z \operatorname{Im} w} \varepsilon_{\bar{w}} \hat{\star} \varepsilon_{z}
$$

and

$$
\hat{\mathcal{S}}_{w}-\hat{\mathcal{S}}_{z}=(w-z) \hat{\mathcal{S}}_{w} \hat{\star} \hat{\mathcal{S}}_{z}+(\bar{z}-\bar{w}) \hat{\mathcal{R}}_{w} \hat{\star} \mathcal{E}_{z}
$$

Proof. If we write $X=z_{w}-z_{z}-(w-z) z_{w} \hat{\star} z_{z}$, it is easy to see that $X \hat{\star}(T-z 1)=0$, and hence

$$
X=X \hat{\star} \mathcal{E}_{z}=\left(1-\frac{i(w-z)}{2 \operatorname{Im} z}\right) z_{w} \hat{\star} \mathcal{E}_{z}-\frac{i}{2 \operatorname{Im} z} \mathcal{E}_{z}
$$

However, in addition $(T-w 1) \hat{\star} X=0$, and hence

$$
X=\mathcal{E}_{\bar{w}} \hat{\star} X=\left(1-\frac{i(w-z)}{2 \operatorname{Im} z}\right) \mathcal{E}_{\bar{w}} \hat{\star} z_{w} \hat{\star} \varepsilon_{z}-\frac{i}{2 \operatorname{Im} z} \mathcal{E}_{\bar{w}} \hat{\star} \mathcal{E}_{z}
$$

Since $z_{\bar{w}} \hat{\star} \varepsilon_{\bar{w}}=-\frac{i}{2 \operatorname{Im} w} \varepsilon_{\bar{w}}$, we deduce that

$$
X=\frac{i}{2 \operatorname{Im} w}\left(1-\frac{i(w-z)}{2 \operatorname{Im} z}\right) \varepsilon_{\bar{w}} \hat{\star} \mathcal{E}_{z}-\frac{i}{2 \operatorname{Im} z} \mathcal{E}_{\bar{w}} \hat{\star} \mathcal{E}_{z}=\frac{\bar{w}-\bar{z}}{4 \operatorname{Im} z \operatorname{Im} w} \varepsilon_{\bar{w}} \hat{\star} \varepsilon_{z}
$$

as required. Similarly, if we write $Y=\hat{\mathcal{S}}_{w}-\hat{\mathcal{S}}_{z}-(w-z) \hat{\mathcal{S}}_{w} \hat{\star} \hat{\mathcal{S}}_{z}$, then we have $Y \hat{\star}(T-z 1)=0$, and hence

$$
Y=Y \hat{\star} \mathcal{E}_{z}=\hat{\mathcal{S}}_{w} \hat{\star} \mathcal{E}_{z}=(\bar{z}-\bar{w}) \hat{\mathcal{R}}_{w} \hat{\star} \mathcal{E}_{z}
$$

as required.

Thus $\mathcal{Z}_{z}$ and $\hat{\mathcal{S}}$ satisfy extended resolvent formulae, with the deficiency distributions $\mathcal{E}_{z}$ again recording the amount by which they fail to satisfy standard resolvent identities. 
Proposition 7.2. As functions from $\mathbb{C} \backslash \mathbb{R}$ to $D_{b}^{\prime}$, the maps $\mathcal{R}, \hat{\mathcal{R}}, \mathcal{S}, \hat{\mathcal{S}}$, $Z$ and $\mathcal{E}$ are all norm-continuous.

Proof. From the resolvent formulae of the previous result, we see that

$$
\begin{gathered}
\left\|z_{w}-z_{z}\right\| \leq|w-z|\left(\frac{4}{|\operatorname{Im} w \operatorname{Im} z|}+\frac{1}{4|\operatorname{Im} w \operatorname{Im} z|}\right) \\
\left\|\hat{\mathcal{S}}_{w}-\hat{\mathcal{S}}_{z}\right\| \leq|w-z|\left(\frac{4}{|\operatorname{Im} w \operatorname{Im} z|}+\frac{1}{(\operatorname{Im} w)^{2}}\right)
\end{gathered}
$$

for all $w, z \in \mathbb{C} \backslash \mathbb{R}$, and hence we deduce that both $z$ and $\hat{\mathcal{S}}$ are norm-continuous. Since both $\mathcal{S}$ and $\mathcal{E}$ can be expressed easily in terms of $z$ and $\hat{\mathcal{S}}$, we deduce that $\mathcal{S}$ and $\mathcal{E}$ are also norm-continuous.

Since $\mathcal{R}_{\bar{z}}=\mathcal{R}_{z}$, we deduce that

$$
\mathcal{S}_{\bar{z}}=\mathcal{R}_{\bar{z}} \hat{\star}(T-z 1)=\mathcal{R}_{z} \hat{\star}(T-z 1)=\mathcal{S}_{z}-(z-\bar{z}) \mathcal{R}_{z}
$$

which implies that

and, similarly,

$$
\mathcal{R}_{z}=-\frac{i}{2 \operatorname{Im} z}\left[\mathcal{S}_{z}-\mathcal{S}_{\bar{z}}\right]
$$

$$
\hat{\mathcal{R}}_{z}=-\frac{i}{2 \operatorname{Im} z}\left[\hat{\mathcal{S}}_{z}-\hat{\mathcal{S}}_{\bar{z}}\right]
$$

and these identities show that $\mathcal{R}$ and $\hat{\mathcal{R}}$ are also norm-continuous.

We can obtain more detailed information about the norm-continuity of each of these distributions, for example by deriving inequalities such as

$$
\left\|\mathcal{R}_{w}-\mathcal{R}_{z}\right\| \leq \frac{2(|\operatorname{Im} w|+|\operatorname{Im} z|)}{(\operatorname{Im} w \operatorname{Im} z)^{2}}|z-w|
$$

for all $z, w \in \mathbb{C} \backslash \mathbb{R}$, but the calculations are lengthy and do not provide additional information of use to us.

Proposition 7.3. As a function from $\mathbb{C} \backslash \mathbb{R} \rightarrow D_{b}^{\prime}$, Z and $\hat{\mathcal{S}}$ are both partially norm-differentiable, with

$$
\begin{aligned}
& \frac{\partial}{\partial x} z_{z}=z_{z} \hat{\star} z_{z}+\frac{1}{4(\operatorname{Im} z)^{2}} \mathcal{E}_{\bar{z}} \hat{\star} \mathcal{E}_{z} \\
& \frac{\partial}{\partial y} z_{z}=i z_{z} \hat{\star} z_{z}-\frac{i}{4(\operatorname{Im} z)^{2}} \mathcal{E}_{\bar{z}} \hat{\star} \mathcal{E}_{z} \\
& \frac{\partial}{\partial x} \hat{\mathcal{S}}_{z}=\hat{\mathcal{S}}_{z} \hat{\star} \hat{\mathcal{S}}_{z}-\hat{\mathcal{R}}_{z} \hat{\star} \mathcal{E}_{z} \\
& \frac{\partial}{\partial y} \hat{\mathcal{S}}_{z}=i \hat{\mathcal{S}}_{z} \hat{\star} \hat{\mathcal{S}}_{z}+i \hat{\mathcal{R}}_{z} \hat{\star} \mathcal{E}_{z}
\end{aligned}
$$


Proof. Special cases of the extended resolvent formula tell us that

$$
\begin{aligned}
& z_{\xi+i y}-z_{x+i y}=(\xi-x) z_{\xi+i y} \hat{\star} z_{x+i y}+\frac{\xi-x}{4 y^{2}} \varepsilon_{\xi-i y} \hat{\star} \mathcal{E}_{x+i y} \\
& z_{x+i \eta}-z_{x+i y}=i(\eta-y) z_{x+i \eta} \hat{\star} z_{x+i y}-\frac{i(\eta-y)}{4 \eta y} \varepsilon_{x+i \eta} \hat{\star} \varepsilon_{x+i y}
\end{aligned}
$$

which, together with the norm-continuity of $Z$ and $\mathcal{E}$, tell us that $Z$ is partially norm-differentiable, with the partial derivatives as indicated. The derivatives of $\hat{\delta}$ are obtained similarly.

This shows definitely that $\mathcal{Z}$ and $\hat{\mathcal{S}}$ are not norm-analytic, since

$$
\begin{aligned}
\left(\frac{\partial}{\partial x}+i \frac{\partial}{\partial y}\right) z_{z} & =\frac{1}{2(\operatorname{Im} z)^{2}} \mathcal{E}_{\bar{z}} \hat{\star} \mathcal{E}_{z} \\
\left(\frac{\partial}{\partial x}+i \frac{\partial}{\partial y}\right) \hat{\mathcal{S}}_{z} & =-2 \hat{\mathcal{R}}_{z} \hat{\star} \mathcal{E}_{z}
\end{aligned}
$$

additionally, since $\mathcal{S}=2 \mathcal{Z}-\hat{\mathcal{S}}$, we can show that

$$
\left(\frac{\partial}{\partial x}+i \frac{\partial}{\partial y}\right) \mathcal{S}_{z}=\left[2 \hat{\mathcal{R}}_{z}+\frac{1}{(\operatorname{Im} z)^{2}} \mathcal{E}_{\bar{z}}\right] \hat{\star} \mathcal{E}_{z}=\left[2 \mathcal{R}_{z}-\frac{1}{(\operatorname{Im} z)^{2}} \mathcal{E}_{\bar{z}}\right] \hat{\star} \mathcal{E}_{z}
$$

and again notice that $\mathcal{S}$ is not analytic.

Although $Z$ is, at first sight, the best candidate for the resolvent function, and we have now seen that $z$ does not have the required analyticity property, it is still possible that we have not yet found the right resolvent function. Any distribution of the form

$$
\mathcal{S}_{z}^{Z}=Z_{z}+\mathcal{E}_{\bar{z}} \hat{\star} Z \hat{\star} \mathcal{E}_{z}, \quad z \in \mathbb{C} \backslash \mathbb{R},
$$

where $Z=\bar{Z} \in D_{b}^{\prime}$, would be a valid two-sided resolvent function for $T$ with $\overline{\mathcal{S}_{z}^{Z}}=\mathcal{S}_{\bar{z}}^{Z}$, and it is possible that a distribution $Z$ could be found for which $\mathcal{S}^{Z}$ was an analytic function. To investigate this, we need to determine the differentiability properties of $\mathcal{E}$.

Proposition 7.4. For any $w, z \in \mathbb{C} \backslash \mathbb{R}$ we have

$$
\begin{aligned}
\mathcal{E}_{w}-\mathcal{E}_{w} \hat{\star} \mathcal{E}_{z} & =(w-z) \mathcal{E}_{w} \hat{\star} \hat{\mathcal{S}}_{z} \\
\mathcal{E}_{w}-\mathcal{E}_{z} & =(w-z) \mathcal{E}_{w} \hat{\star} \hat{\mathcal{S}}_{z}+(\bar{w}-\bar{z}) \overline{\hat{\mathcal{S}}_{w}} \hat{\star} \mathcal{E}_{z}
\end{aligned}
$$

Proof. If we write $X=\mathcal{E}_{w}-\mathcal{E}_{z}-(w-z) \mathcal{E}_{w} \hat{\star} \hat{\mathcal{S}}_{z}$, it is clear that $X \hat{\star}(T-$ $z 1)=0$, and hence

$$
X=X \hat{\star} \mathcal{E}_{z}=\mathcal{E}_{w} \hat{\star} \mathcal{E}_{z}-\mathcal{E}_{z}
$$


Simplifying this equation we obtain

$$
\mathcal{E}_{w}-\mathcal{E}_{w} \hat{\star} \mathcal{E}_{z}=(w-z) \mathcal{E}_{w} \hat{\star} \hat{\mathcal{S}}_{z}
$$

Hence

$$
-X=\mathcal{E}_{z}-\mathcal{E}_{w} \hat{\star} \mathcal{E}_{z}=\overline{\mathcal{E}_{z}-\mathcal{E}_{z} \hat{\star} \mathcal{E}_{w}}=-(\bar{w}-\bar{z}) \overline{\hat{\mathcal{S}}_{w}} \hat{\star} \mathcal{E}_{z}
$$

which implies that

$$
\mathcal{E}_{w}-\mathcal{E}_{z}=(w-z) \mathcal{E}_{w} \hat{\star} \hat{\mathcal{S}}_{z}+(\bar{w}-\bar{z}) \overline{\hat{\mathcal{S}}_{w}} \hat{\star} \mathcal{E}_{z}
$$

as required.

As a consequence of these results, we obtain the following. The proof is similar to previous ones.

Proposition 7.5. The function $\mathcal{E}$ is partially norm-differentiable from $\mathbb{C} \backslash \mathbb{R}$ to $D_{b}^{\prime}$, and

$$
\begin{aligned}
& \frac{\partial}{\partial x} \mathcal{E}_{z}=\mathcal{E}_{z} \hat{\star} \hat{\mathcal{S}}_{z}+\overline{\hat{\mathcal{S}}_{z}} \hat{\star} \mathcal{E}_{z} \\
& \frac{\partial}{\partial y} \mathcal{E}_{z}=i \mathcal{E}_{z} \hat{\star} \hat{\mathcal{S}}_{z}-i \overline{\hat{\mathcal{S}}_{z}} \hat{\star} \mathcal{E}_{z}
\end{aligned}
$$

As a consequence we deduce that

$$
\begin{aligned}
& \left(\frac{\partial}{\partial x}+i \frac{\partial}{\partial y}\right) \mathcal{E}_{z}=2 \overline{\hat{S}}_{z} \hat{\star} \mathcal{E}_{z} \\
& \left(\frac{\partial}{\partial x}+i \frac{\partial}{\partial y}\right) \mathcal{E}_{\bar{z}}=2 \mathcal{E}_{\bar{z}} \hat{\star} \hat{\mathcal{S}}_{\bar{z}}
\end{aligned}
$$

and this implies that, in order to find a working resolvent function $\mathcal{S}^{Z}$, we need to find an operator $Z=\bar{Z} \in D_{b}^{\prime}$ such that

$$
\begin{aligned}
\left(\frac{\partial}{\partial x}+i \frac{\partial}{\partial y}\right) \mathcal{S}_{z}^{Z} & =\frac{1}{2(\operatorname{Im} z)^{2}} \mathcal{E}_{\bar{z}} \hat{\star} \mathcal{E}_{z}+2 \mathcal{E}_{\bar{z}} \hat{\star}\left(Z \hat{\star} \hat{\mathcal{S}}_{z}+\overline{\hat{\mathcal{S}}_{\bar{z}}} \hat{\star} Z\right) \hat{\star} \mathcal{E}_{z} \\
& =\frac{1}{2(\operatorname{Im} z)^{2}} \mathcal{E}_{\bar{z}} \hat{\star} \mathcal{E}_{z}-8 i \operatorname{Im} z \mathcal{E}_{\bar{z}} \hat{\star}\left(Z \hat{\star} \hat{R}_{z}+\overline{\hat{R}_{\bar{z}}} \hat{\star} Z\right) \hat{\star} \mathcal{E}_{z}=0
\end{aligned}
$$

for all $z \in \mathbb{C} \backslash \mathbb{R}$, a task that seems moderately unlikely!

In conclusion, therefore, we must conclude that the distribution-valued approach to spectral theory does not provide us with any new spectral calculus for operators for which one did not already exist, which is a pity. 


\section{$\S 8 . \quad$ Conclusions}

Were the Hansen approach to spectral theory to deliver a resolvent-based spectral theory for symmetric operators (and not just for essentially self-adjoint ones), the complications detailed above would be wholly justified. As it stands, however, this has not happened. There is, however, the possibility that classes of more appropriate distributions do exist and could be discovered. Until that time, it seems that the non-self-adjoint operators are better considered by means of positive operator-valued measures. Since these are the outcome of an operational approach to quantum mechanics they emerge from the theory of measurements quite naturally.

Nonetheless we reiterate something we said in the introduction: the ideas of Hansen are original and interesting. It seems as if nature is providing us with a new insight into the connection between classical and quantum mechanics known as Weyl quantization, an insight but faintly discerned, and clothed in formidable technical formalism. But as mathematics is the science that draws necessary conclusions, the search for effective distribution classes should not be abandoned.

\section{Appendix}

Here we list the correspondences between the conventions of Hansen's paper, [9], and those used in our book on quantization, DHS [5]. In what follows, Hansen's notation is in the left-hand column, and ours is on the right.

Hansen uses the symbol $E$ to denote phase space, as opposed to $\Pi$ in DHS, and there are different conventions for the measure on phase space. In DHS the standard Lebesgue measure $A$ is used, while Hansen normalizes the Haar measure to obtain $\nu=\frac{1}{2 \pi} A$. The following equations set out the identifications between Hansen's notation and that of DHS.

$$
\begin{array}{rll}
\iint_{E} f d \nu & \Leftrightarrow & \frac{1}{2 \pi} \iint_{\Pi} f(x) d A(x) \\
(f \mid g)_{2} & \Leftrightarrow & \frac{1}{2 \pi}\langle f, g\rangle \\
\|f\|_{2} & \Leftrightarrow & \frac{1}{\sqrt{2 \pi}}\|f\| \\
L^{2}(E, d \nu)=H & \Leftrightarrow & L^{2}(\Pi)
\end{array}
$$

In this paper, an integral without an explicit measure is understood to be using the normalized measure $d \nu$. 
The symbol for the Moyal product here has been chosen so that it coincides with that of DHS. The notation is now more or less standard. ${ }^{5}$ :

Unfortunately, these maps are not identical, since different conventions have been used. To complete the connection, we define the map $\mathcal{R}: L^{2}(E, d \nu) \rightarrow$ $L^{2}(E, d \nu)$ by setting $(\mathcal{R} f)(x)=f(\sqrt{2} x)$, for then

$$
\mathcal{R}(f \circ g)=\mathcal{R} f \star \mathcal{R} g
$$

and now the inequalities

(A.4) $\|f \circ g\|_{2} \leq \frac{1}{2}\|f\|_{2}\|g\|_{2} \quad \Leftrightarrow \quad\|f \star g\| \leq \frac{1}{\sqrt{2 \pi}}\|f\| \cdot\|g\|$

demonstrating the joint continuity of the Moyal products are consistent.

It is now possible to make the necessary identifications between the sequences of special functions $\left(a_{m, n}\right)$ and the special Hermite functions $\left(\Phi_{m, n}\right)$ used in DHS. The fundamental identification is

$$
\mathcal{R} a_{m, n}=\phi_{n, m}
$$

which leads to the following correspondences concerning the function $\Omega$ :

$$
\begin{array}{rrrl}
\text { (A.6.a) } & \Omega=a_{0}=a_{0,0} & \Leftrightarrow & \phi_{0,0} \\
\text { (A.6.b) } & \|\Omega\|_{2}=\sqrt{2} & \Leftrightarrow & \left\|\phi_{0,0}\right\|=\sqrt{2 \pi} \\
\text { (A.6.c) } & \Omega \circ f \circ \Omega=\frac{1}{2}(\Omega \mid f)_{2} \Omega & \Leftrightarrow & \phi_{0,0} \star f \star \phi_{0,0}=\frac{1}{2 \pi}\left\langle\phi_{0,0}, f\right\rangle \phi_{0,0} \\
\text { (A.6.d) } & \Omega \circ \Omega=\Omega=\bar{\Omega} & \Leftrightarrow & \phi_{0,0} \star \phi_{0,0}=\phi_{0,0}=\overline{\phi_{0,0}} \\
\text { (A.6.e) } & I_{\Omega}\left(\text { or } H_{\Omega}\right) & \Leftrightarrow & \mathcal{H} \\
\text { (A.6.f) } & \bar{f} \circ g=\frac{1}{2}(f \mid g)_{2} \Omega & \Leftrightarrow & \bar{f} \star g=\frac{1}{2 \pi}\langle f, g\rangle \phi_{0,0}
\end{array}
$$

More general correspondences between these special functions can be seen now:

$$
\begin{array}{rrrl}
\text { (A.7.a) }\left(a_{m, n} \mid a_{j, k}\right)_{2}=2 \delta_{m j} \delta_{n k} & \Leftrightarrow & \left\langle\phi_{m, n}, \phi_{j, k}\right\rangle=2 \pi \delta_{m j} \delta_{n k} \\
\text { (A.7.b) } \quad a_{m, n}=a_{m} \circ \overline{a_{n}} & \Leftrightarrow \quad \phi_{m, n}=\phi_{0, m} \star \overline{\phi_{0, n}} \\
\text { (A.7.c) } & \overline{a_{m, n}}=a_{n, m} & \Leftrightarrow & \phi_{m, n}=\phi_{n, m} \\
\text { (A.7.d) } & a_{m, n} \circ a_{j, k}=\delta_{n, j} a_{m, k} & \Leftrightarrow & \phi_{m, n} \star \phi_{j, k}=\delta_{m, k} \phi_{j, n}
\end{array}
$$

\footnotetext{
${ }^{5}$ Hansen uses the symbol $\circ$ here, but we will retain the now-standard form. The twisted convolution is not explicitly used here, but we note that Hansen uses the symbol $\times$ for it while the symbol $\circ$ is used in DHS.
} 
In this paper, we have chosen to use the notation of Hansen (so that the results we obtain are most readily comparable with those in [9]) with one key exception. Hansen's choice of the symbol $\circ$ to represent the Moyal product is not the accepted standard notation, and hence we have replaced that symbol by the star symbol $\star$. This does mean, however, that the operator $\star$ of this paper and the operator $\star$ in DHS [5] are not the same (although, as we see, closely connected through elementary changes of convention in the matter of inner products and so forth).

Remark. Weyl quantization is only mentioned in passing in Hansen's paper, but it is certainly there. Were it not, the spectral theory in phase space would not be of interest for the spectral theory of the Schrödinger representation. It can be extracted as follows. We refer particularly to subsection 13.2.3, Quantization in Phase Space, of [5]. There we introduce the left regular representation $\mathcal{R}$, Theorem 13.10, and show that it is a ${ }^{\star}$-representation of $\left(L^{2}(\Pi), \star,-\right)$ which is unitarily equivalent to Weyl quantization via the map $\mathcal{V}$, see equation (13.2.13a). It follows that the representation $\left.\hat{\pi}\right|_{H}$ of $H$ is unitarily equivalent to Weyl quantization.

We also note that the connection to Weyl quantization is implicit in Section 2.3 of Folland, [6]. The equivalence of $\hat{\pi}$ to a Schrödinger representation of $D$ into $L^{2}(\mathbb{R})$, of infinite multiplicity, is found in Theorem 2 of Várilly and GraciaBondía, [26].

\section{Acknowledgments}

Much of the work on this paper was done while one of us (M.A.H.) was a Fellow at Sidney Sussex College, Cambridge. One of us (D.A.D.) wishes to thank Dr. D. Crosby and Professor J. D. Silver for extending him the hospitality of Oxford University in general and the EBIT Lab in particular. The authors are indebted to the anonymous referee for providing additional references, correcting some misprints, and suggesting an improved overview of the material.

\section{References}

[1] J.-P. Antoine, A. Inoue and C. Trapani, Partial *-algebras of closable operators: a review, Rev. Math. Phys. 8 (1996), no. 1, 1-42.

[2] M. A. Antonets, Classical limit of the Weyl quantization, Teoret. Mat. Fiz. 38 (1979), no. $3,331-344$. 
[3] I. Daubechies, On the distributions corresponding to bounded operators in the Weyl quantization, Comm. Math. Phys. 75 (1980), no. 3, 229-238.

[4] D. A. Dubin and M. A. Hennings, Quantum mechanics, algebras and distributions, Pitman Research Notes in Mathematics 238, Longman Sci. Tech., Harlow, 1990.

[5] D. A. Dubin, M. A. Hennings and T. B. Smith, Mathematical aspects of Weyl quantization and phase, World Sci. Publishing, River Edge, NJ, 2000.

[6] G. B. Folland, Harmonic analysis in phase space, Ann. of Math. Stud., 122, Princeton Univ. Press, Princeton, NJ, 1989.

[7] V. Gayral, J. M. Gracia-Bondía, B. Iochum, T. Schücker and J. C. Várilly, Moyal planes are spectral triples, Comm. Math. Phys. 246 (2004), no. 3, 569-623.

[8] M. Gerstenhaber and S. D. Schack, Algebras, bialgebras, quantum groups, and algebraic deformations, in Deformation theory and quantum groups with applications to mathematical physics (Amherst, MA, 1990), 51-92, Contemp. Math., 134, Amer. Math. Soc., Providence, RI.

[9] F. Hansen, The Moyal product and spectral theory for a class of infinite-dimensional matrices, Publ. Res. Inst. Math. Sci. 26 (1990), no. 6, 885-933.

[10] L. Hörmander, Pseudo-differential operators and hypoelliptic equations, in Singular integrals (Proc. Sympos. Pure Math., Vol. X, Chicago, Ill., 1966), 138-183, Amer. Math. Soc., Providence, RI, 1967.

[11] The Weyl calculus of pseudodifferential operators, Comm. Pure Appl. Math. 32 (1979), no. 3, 360-443.

[12] J.-B. Kammerer, L'algèbre des opérateurs de multiplication du star-produit de $\mathbf{R}^{2 n}, \mathrm{C}$. R. Acad. Sci. Paris Sér. I Math. 298 (1984), no. 4, 59-62.

[13] Analysis of the Moyal product in a flat space, J. Math. Phys. 27 (1986), no. 2, $529-535$.

[14] J. J. Kohn and L. Nirenberg, An algebra of pseudo-differential operators, Comm. Pure Appl. Math. 18 (1965), 269-305.

[15] Y. Kosmann-Schwarzbach, Tribute to André Lichnerowicz, Notices of the A. M. S., 56, Number 2, February 2009.

[16] N. P. Landsman, Mathematical topics between classical and quantum mechanics, Springer, New York, 1998.

[17] J. E. Moyal, Quantum mechanics as a statistical theory, Proc. Cambridge Philos. Soc. 45 (1949), 99-124.

[18] J. von Neumann, Die Eindeutigkeit der Schrödingerschen Operatoren, Math. Ann. 104 (1931), no. 1, 570-578.

[19] Mathematical foundations of quantum mechanics, Translated by Robert T. Beyer, Princeton Univ. Press, Princeton, 1955.

[20] J. C. T. Pool, Mathematical aspects of the Weyl correspondence, J. Mathematical Phys. 7 (1966), 66-76

[21] M. A. Rieffel, Deformation quantization for actions of $\mathbf{R}^{d}$, Mem. Amer. Math. Soc. 106 (1993), no. 506, $\mathrm{x}+93$ pp.

[22] K. Schmüdgen, Unbounded operator algebras and representation theory, Birkhäuser, Basel, 1990.

[23] L. Schwartz, Théorie des Distributions I, II, Hermann \& Cie. Paris, 1950-1951.

[24] M. A. Shubin, Pseudodifferential operators and spectral theory, Translated from the 1978 Russian original by Stig I. Andersson, Second edition, Springer, Berlin, 2001.

[25] S. Thangavelu, Lectures on Hermite and Laguerre expansions, Princeton Univ. Press, Princeton, NJ, 1993

[26] J. M. Gracia-Bondía and J. C. Várilly, Algebras of distributions suitable for phase-space quantum mechanics. I, II, J. Math. Phys. 29 (1988), no. 4, 869-879 and 880-887.

[27] H. Weyl, Quantenmechanik und Gruppentheorie, Zeitschrift für Physik, 46 (1927), 1-46.

[28] - The theory of groups and quantum mechanics, Dover, New York, 1931.

[29] E. P. Wigner, On the quantum correction for thermodynamic equilibrium, Phys. Rev. 40 (1932), 749-759. 